\title{
A Novel Multi-parameter Family of Quantum Systems with Partially Broken $\mathcal{N}$-fold Supersymmetry
}

\author{
Artemio González-Lóped* and Toshiaki Tanakdđ \\ Departamento de Física Teórica II, Facultad de Ciencias Físicas, \\ Universidad Complutense, 28040 Madrid, Spain
}

(Dated: January 31, 2005; revised June 2, 2005)

\begin{abstract}
We develop a systematic algorithm for constructing an $\mathcal{N}$-fold supersymmetric system from a given vector space invariant under one of the supercharges. Applying this algorithm to spaces of monomials, we construct a new multi-parameter family of $\mathcal{N}$-fold supersymmetric models, which shall be referred to as "type C". We investigate various aspects of these type C models in detail. It turns out that in certain cases these systems exhibit a novel phenomenon, namely, partial breaking of $\mathcal{N}$-fold supersymmetry.
\end{abstract}

PACS numbers: 03.65.Fd, 03.65.Ge, 11.30.Pb, 11.30.Na

Keywords: quantum mechanics, quasi(-exact) solvability, $\mathcal{N}$-fold supersymmetry, intertwining relation, dynamical symmetry breaking

*email: artemio@fis.ucm.es

$\dagger$ email: ttanaka@mail.tku.edu.tw 


\section{INTRODUCTION}

A significant progress in scientific research has often been achieved through the unification of seemingly unrelated concepts. Recently, three different theoretical developments were unified in the framework of $\mathcal{N}$-fold supersymmetry [1, 2], namely: i) isospectral transformations, traced back to the work of Darboux in the late nineteenth century [3] (see also Ref. [4] and references therein), and their higher-derivative generalizations first formulated in Ref. [5], ii) quasi-exact solvability in one-dimensional quantum mechanical systems [6] (see also Ref. 7] and references therein), and iii) a particular class of nonlinear superalgebras. The characteristic feature of $\mathcal{N}$-fold supersymmetry, which distinguishes it from other nonlinear extensions of ordinary supersymmetry such as parasupersymmetry [8, 9, 10] and fractional supersymmetry [11], is the fact that anticommutators of fermionic operators are polynomials of degree (at most) $\mathcal{N}$ in bosonic operators. Usually, $\mathcal{N}$-fold supercharges are represented by $\mathcal{N}$ th-order linear differential operators (see references in Ref. [12]).

The unification mentioned in the previous paragraph comes about schematically as follows. An $\mathcal{N}$-fold supersymmetric quantum system always yields, by definition, a pair of isospectral Hamiltonians $H^{ \pm}$. These operators are automatically quasi-solvable, since they preserve the kernel of (the bosonic part of) the respective supercharges $Q^{ \pm}$. Finally, the anticommutator $\left\{Q^{+}, Q^{-}\right\}$is a polynomial of degree (at most) $\mathcal{N}$ in the superHamiltonian $\mathbf{H}$, which accounts for the polynomial character of the superalgebra closed by $\mathbf{H}$ and $Q^{ \pm}$. Although these ideas are conceptually simple, the direct construction of $\mathcal{N}$-fold supersymmetric models becomes rather unwieldy for large $\mathcal{N}$, since intertwining relations with respect to a higher-order differential operator are quite complicated. Indeed, type A $\mathcal{N}$-fold supersymmetry [13, 14] is virtually the only known class for which most of the aspects mentioned above are well understood for arbitrary $\mathcal{N}$.

One of the characteristic features of type A $\mathcal{N}$-fold supersymmetric models is that, after a suitable gauge transformation, their supercharges leave invariant the space of polynomials in one variable of degree less than $\mathcal{N}$. In our previous paper [15] we obtained a new family of $\mathcal{N}$-fold supersymmetric models, the so-called type $\mathrm{B}$, by considering what is probably the simplest deformation of the type A $\mathcal{N}$-fold supercharge. Although the construction presented in this reference was rather $a d$ hoc and of a purely analytic nature, the resulting type B supercharges (after an appropriate gauge transformation) preserve a finite-dimensional linear space of monomial type. These results strongly suggest that it can be of great advantage to base the construction of $\mathcal{N}$-fold supersymmetric models not on a specific form of the supercharge, but rather on the finite-dimensional linear space invariant under one of the supercharges, say $Q^{-}$.

In this article we show that this idea is indeed feasible, by developing a systematic algorithm for constructing an $\mathcal{N}$-fold supersymmetric system starting from the knowledge of the $\mathcal{N}$-dimensional linear space of functions left invariant by a suitable gauge transform of the supercharge $Q^{-}$. One of the main advantages of this method is that it completely bypasses the "hard" calculation of the intertwining relations, which makes it ideally suited when $\mathcal{N}$ is not fixed a priori. We then use our algorithm to derive the $\mathcal{N}$-fold supersymmetric systems arising when one chooses as the starting point in the construction what is perhaps the simplest possibility, namely a space of monomials. According to Post and Turbiner [16], there exist essentially three inequivalent finite-dimensional monomial spaces preserved by a nonzero second-order linear differential operator. It turns out that two of them lead to the already known $\mathcal{N}$-fold supersymmetric models of types $\mathrm{A}$ and $\mathrm{B}$. The remaining one 
yields a new type of supersymmetry, referred to as type $C$ in what follows, which is one of the main contributions of this paper. The type $\mathrm{C} \mathcal{N}$-fold supercharge is in a sense the most general supercharge of arbitrary order $\mathcal{N}$, since it formally reduces to all previously known instances, namely, the type A and B supercharges. We fully classify the type C models, finding in particular explicit formulas for their potentials, and further analyze the main properties of these models, such as shape-invariance, associated polynomial families of Bender-Dunne type, and the resulting $\mathcal{N}$-fold superalgebras.

The article is organized as follows. In the next section, after reviewing briefly the concepts of $\mathcal{N}$-fold supersymmetry and quasi-solvability, as well as their mutual relationship, we outline the general procedure for constructing an $\mathcal{N}$-fold supersymmetric system starting from a given finite-dimensional linear space of functions. The connection with the construction in Refs. [14, 15] of type A and B models is also briefly discussed. In Section [II we apply the general method to the simple case in which the given linear space of functions is of monomial type, obtaining a new multi-parameter family of $\mathcal{N}$-fold supersymmetry, namely type $\mathrm{C}$. We investigate the general properties of type $\mathrm{C}$ models, such as the shape invariance between the partner Hamiltonians, the structure of the solvable sectors, and the symmetry transformations which preserve the potential form. Using the invariance under these symmetry transformations, we completely classify the type $\mathrm{C}$ models in Section IV. We find that there are essentially four inequivalent nontrivial types of potentials. The normalizability of the solvable sectors is also briefly examined in this section. In Section $\nabla$, we study the polynomial families of Bender-Dunne type associated with the $\mathcal{N}$-fold supersymmetric models of type $\mathrm{C}$. We prove that to each type $\mathrm{C}$ model one can associate two polynomial families which, in contrast to their type A counterparts, are always weakly orthogonal. They also exhibit a novel feature, namely their dependence on two integer parameters. It is also shown that the polynomial part of the type $\mathrm{C} \mathcal{N}$-fold superalgebra can be expressed as a product of two critical polynomials belonging to each of the above families. A few examples are also exhibited explicitly at the end of this section. Finally, in Section VI we discuss several general aspects of type $\mathrm{C}$ models, such as the partial breaking of $\mathcal{N}$-fold supersymmetry, and briefly discuss future developments suggested by the present work.

\section{II. $\mathcal{N}$-FOLD SUPERSYMMETRY AND QUASI-SOLVABILITY}

In this section we shall review the concept of $\mathcal{N}$-fold supersymmetry in one-dimensional quantum mechanics, making special emphasis on its connections with the recently introduced notion of quasi-solvability. We shall then outline a general algorithmic procedure for constructing $\mathcal{N}$-fold supersymmetric models starting from an $\mathcal{N}$-dimensional linear space invariant under the action of a second-order linear differential operator.

Let $q$ denote a bosonic coordinate, and let $\psi$ and $\psi^{\dagger}$ be fermionic coordinates satisfying

$$
\{\psi, \psi\}=\left\{\psi^{\dagger}, \psi^{\dagger}\right\}=0, \quad\left\{\psi, \psi^{\dagger}\right\}=1 .
$$

Given a monic $\mathcal{N}$ th-order linear differential operator

$$
P_{\mathcal{N}}=\partial_{q}^{\mathcal{N}}+\sum_{k=0}^{\mathcal{N}-1} w_{k}(q) \partial_{q}^{k},
$$

we introduce the $\mathcal{N}$-fold supercharges $Q_{\mathcal{N}}^{ \pm}$by

$$
Q_{\mathcal{N}}^{-}=P_{\mathcal{N}^{-}}^{-\dagger}, \quad Q_{\mathcal{N}}^{+}=P_{\mathcal{N}}^{+} \psi
$$


where the operators $P_{\mathcal{N}}^{ \pm}$are defined by

$$
P_{\mathcal{N}}^{-}=P_{\mathcal{N}}, \quad P_{\mathcal{N}}^{+}=(-1)^{\mathcal{N}} P_{\mathcal{N}}^{t}
$$

The superscript ${ }^{t}$ in the latter equation denotes the transposed operator defined by $A^{t}=$ $\left(A^{\dagger}\right)^{*}$, where the star denotes complex conjugation. If, as is usually the case, all the coefficients $w_{k}$ in Eq. (II.2) are real, then $P_{\mathcal{N}}^{t}$ obviously coincides with $P_{\mathcal{N}}^{\dagger}$. The nilpotency of the fermionic variables $\psi$ and $\psi^{\dagger}$ implies that

$$
\left\{Q_{\mathcal{N}}^{-}, Q_{\mathcal{N}}^{-}\right\}=\left\{Q_{\mathcal{N}}^{+}, Q_{\mathcal{N}}^{+}\right\}=0
$$

We define a superHamiltonian $\mathbf{H}$ by

$$
\mathbf{H}=H^{-} \psi \psi^{\dagger}+H^{+} \psi^{\dagger} \psi
$$

where

$$
H^{ \pm}=-\frac{1}{2} \partial_{q}^{2}+V^{ \pm}(q)
$$

is a pair of scalar Hamiltonians. An $\mathcal{N}$-fold supersymmetric model is a triple $\left(\mathbf{H}, Q_{\mathcal{N}}^{+}, Q_{\mathcal{N}}^{-}\right)$ such that the supercharges $Q_{\mathcal{N}}^{ \pm}$commute with $\mathbf{H}$, namely

$$
\left[Q_{\mathcal{N}}^{ \pm}, \mathbf{H}\right]=0
$$

Equation (11.8) is equivalent to the following intertwining relations between the component supercharges $P_{\mathcal{N}}^{ \pm}$and Hamiltonians $H^{ \pm}$:

$$
P_{\mathcal{N}}^{-} H^{-}-H^{+} P_{\mathcal{N}}^{-}=0, \quad P_{\mathcal{N}}^{+} H^{+}-H^{-} P_{\mathcal{N}}^{+}=0
$$

Since the Hamiltonians $H^{ \pm}$are both symmetric under transposition, each one of the relations (II.9) actually follows from the other one by transposition.

Following Ref. [14], we shall say that a differential operator $T$ is weakly quasi-solvable with respect to an $\mathcal{N}$ th-order differential operator $P_{\mathcal{N}}$ of the form (II.2) if it leaves ker $P_{\mathcal{N}}$ invariant. If, in addition, $\operatorname{ker} P_{\mathcal{N}}$ can be explicitly computed we shall simply say that $T$ is quasi-solvable. We shall also use the term quasi-exactly solvable to refer to a quasi-solvable operator whose finite-dimensional invariant space is a subspace of the Hilbert space on which the operator is naturally defined. ${ }^{1}$ A particular class of quasi-solvable operators is that of Lie-algebraic operators, which are polynomials in the generators of a finite-dimensional Lie algebra of first-order differential operators preserving a known finite-dimensional linear space [6, 7, 18, 19].

From Eqs. (II.9) it immediately follows that each one of the component Hamiltonians $H^{ \pm}$ leaves invariant the kernel $\mathcal{V}_{\mathcal{N}}^{ \pm}$of the corresponding operator $P_{\mathcal{N}}^{ \pm}$. Hence both $H^{-}$and $H^{+}$are (weakly) quasi-solvable with respect to the operators $P_{\mathcal{N}}^{-}$and $P_{\mathcal{N}}^{+}$, respectively. Remarkably, the converse is also true. Indeed [1], from a scalar Hamiltonian $H$ weakly quasi-solvable with

\footnotetext{
${ }^{1}$ Unfortunately, the definition of the term "quasi-exactly solvable" in the literature is far from uniform. In this paper we have adopted the terminology recently proposed by one of the authors in Refs. [12, 17].
} 
respect to an $\mathcal{N}$ th-order linear differential operator $P_{\mathcal{N}}$ of the form (II.2) one can construct an $\mathcal{N}$-fold supersymmetric model by taking $P_{\mathcal{N}}^{ \pm}$as in (II.4) and

$$
H^{-}=H, \quad H^{+}=H+\dot{w}_{\mathcal{N}-1}(q),
$$

where the dot denotes derivative with respect to $q$. Thus there is a one-to-one correspondence between $\mathcal{N}$-fold supersymmetric models and weakly quasi-solvable scalar Hamiltonians.

From the above remarks it follows that, given an $\mathcal{N}$-dimensional linear space

$$
\mathcal{V}_{\mathcal{N}}=\left\langle\varphi_{1}(q), \ldots, \varphi_{\mathcal{N}}(q)\right\rangle
$$

and a scalar Hamiltonian $H$ leaving $\mathcal{V}_{\mathcal{N}}$ invariant, one can construct an $\mathcal{N}$-fold supersymmetric model using Eqs. (II.3)-(II.4) and (II.10) and taking as $P_{\mathcal{N}}$ the unique $\mathcal{N}$ th-order linear differential operator (II.2) annihilating $\mathcal{V}_{\mathcal{N}}$, namely

$$
P_{\mathcal{N}}=W\left(\varphi_{1}, \ldots, \varphi_{\mathcal{N}}\right)^{-1}\left|\begin{array}{cccc}
1 & \partial_{q} & \ldots & \partial_{q}^{\mathcal{N}} \\
\varphi_{1} & \dot{\varphi}_{1} & \ldots & \varphi_{1}^{(\mathcal{N})} \\
\vdots & \vdots & \ddots & \vdots \\
\varphi_{\mathcal{N}} & \dot{\varphi}_{\mathcal{N}} & \ldots & \varphi_{\mathcal{N}}^{(\mathcal{N})}
\end{array}\right|
$$

where $W\left(\varphi_{1}, \ldots, \varphi_{\mathcal{N}}\right)$ is the Wronskian of the functions $\varphi_{1}, \ldots, \varphi_{\mathcal{N}}$ spanning $\mathcal{V}_{\mathcal{N}}$ (cf. Refs. 20, 21]). The latter scheme for constructing $\mathcal{N}$-fold supersymmetric models suffers from two major drawbacks, namely: i) an arbitrary $\mathcal{N}$-dimensional linear space (II.11) is not preserved, in general, by any scalar Hamiltonian, and ii) even when this is the case, it may be difficult to find explicitly a Hamiltonian leaving (II.11) invariant.

To overcome these drawbacks, let us slightly generalize the above construction by considering an $\mathcal{N}$-dimensional linear space

$$
\tilde{\mathcal{V}}_{\mathcal{N}}=\left\langle\tilde{\varphi}_{1}(z), \ldots, \tilde{\varphi}_{\mathcal{N}}(z)\right\rangle
$$

and a scalar second-order linear differential operator (not necessarily a Hamiltonian)

$$
-\tilde{H}=A(z) \partial_{z}^{2}+B(z) \partial_{z}+C(z)
$$

leaving $\tilde{\mathcal{V}}_{\mathcal{N}}$ invariant. From these two ingredients one can construct an $\mathcal{N}$-fold supersymmetric model as follows.

Let

$$
\tilde{P}_{\mathcal{N}}=g(z)\left(\partial_{z}^{\mathcal{N}}+\sum_{k=0}^{\mathcal{N}-1} \tilde{w}_{k}(z) \partial_{z}^{k}\right)
$$

denote the most general $\mathcal{N}$ th-order linear differential operator with kernel $\tilde{\mathcal{V}}_{\mathcal{N}}$, where the function $g(z)$ is for the time being undetermined. We shall first construct another secondorder linear differential operator of the form

$$
\tilde{H}^{+}=\tilde{H}-\delta C
$$

satisfying the intertwining relation

$$
\tilde{P}_{\mathcal{N}} \tilde{H}-\tilde{H}^{+} \tilde{P}_{\mathcal{N}}=0
$$


To this end, note that the left-hand side of (II.17) is in general a linear differential operator of order $\mathcal{N}+1$. Equating to zero the coefficients of $\partial_{z}^{\mathcal{N}+1}$ and $\partial_{z}^{\mathcal{N}}$ in this operator we obtain the following two equations for the functions $g$ and $\delta C$ :

$$
\begin{aligned}
\frac{g^{\prime}}{g}= & \frac{\mathcal{N}}{2} \frac{A^{\prime}}{A} \\
\delta C= & \frac{1}{2} \mathcal{N}(\mathcal{N}-2)\left(A^{\prime \prime}-\frac{A^{\prime 2}}{2 A}\right)+\mathcal{N}\left(B^{\prime}-\frac{B A^{\prime}}{2 A}\right) \\
& -A^{\prime} \tilde{w}_{\mathcal{N}-1}-2 A \tilde{w}_{\mathcal{N}-1}^{\prime},
\end{aligned}
$$

where the prime denotes derivative with respect to $z$. When Eqs. (II.18) - (II.19) are satisfied, the l.h.s. of (II.17) is a linear differential operator of order at most $\mathcal{N}-1$ annihilating the $\mathcal{N}$-dimensional space $\tilde{\mathcal{V}}_{\mathcal{N}}$, and hence it vanishes identically.

The last step in our construction consists in applying a change of variable

$$
z=z(q)
$$

and a gauge transformation with gauge factor $\mathrm{e}^{-\mathcal{W}(z)}$, under which

$$
\left.\tilde{H}^{ \pm} \mapsto \mathrm{e}^{-\mathcal{W}(z)} \tilde{H}^{ \pm} \mathrm{e}^{\mathcal{W}(z)}\right|_{z=z(q)} \equiv H^{ \pm}
$$

to simultaneously take $\tilde{H}^{-} \equiv \tilde{H}$ and $\tilde{H}^{+}$to Schrödinger form (II.7). Note that this is certainly possible, since (by construction) $\tilde{H}$ and $\tilde{H}^{+}$differ by a scalar function only. The appropriate change of variable and gauge transformation are determined by [19, 22]

$$
\begin{aligned}
\dot{z}^{2} & =2 A(z) \\
\mathcal{W}^{\prime} & =\frac{1}{2 A}\left(\frac{A^{\prime}}{2}-B\right) .
\end{aligned}
$$

The potentials $V^{ \pm}(q)$ are related to the coefficients of the differential operators $\tilde{H}^{ \pm}$as follows:

$$
V^{ \pm}(q)=-C^{ \pm}+\left.\frac{1}{4 A}\left[B^{2}-A A^{\prime \prime}+\frac{3}{4} A^{2}+2\left(A B^{\prime}-A^{\prime} B\right)\right]\right|_{z=z(q)},
$$

where $C^{-} \equiv C, C^{+}=C^{-}+\delta C$. More explicitly,

$$
\begin{aligned}
V^{ \pm}(q)= & -C+\frac{A^{\prime} \tilde{w}_{\mathcal{N}-1}}{2}+A \tilde{w}_{\mathcal{N}-1}^{\prime}-\frac{1}{2}(\mathcal{N}-1)\left(Q^{\prime}+\frac{A^{\prime \prime}}{2}\right) \\
& +\frac{1}{4 A}\left(Q^{2}+\left(\mathcal{N}^{2}-1\right) \frac{A^{2}}{4}\right) \\
& \pm\left.\left[A \tilde{w}_{\mathcal{N}-1}^{\prime}-\frac{\mathcal{N} Q^{\prime}}{2}+\left(\frac{\mathcal{N} Q}{4 A}+\frac{\tilde{w}_{\mathcal{N}-1}}{2}\right) A^{\prime}\right]\right|_{z=z(q)},
\end{aligned}
$$

where, following Ref. [15], we have set

$$
Q=B+\frac{1}{2}(\mathcal{N}-2) A^{\prime}
$$


From the above construction it immediately follows that the system (II.3) -(II.4) and (II.6), with $H_{\mathcal{N}}^{ \pm}$given by (II.21) and

$$
P_{\mathcal{N}}=\left.\mathrm{e}^{-\mathcal{W}(z)} \tilde{P}_{\mathcal{N}} \mathrm{e}^{\mathcal{W}(z)}\right|_{z=z(q)}
$$

is $\mathcal{N}$-fold supersymmetric. Indeed, the first intertwining relation (II.9) follows by applying the gauge transformation and change of variable (II.21) to the relation (II.17). Note also that (II.26) and the definition of $\tilde{P}_{\mathcal{N}}$ imply that the kernel of the operator $P_{\mathcal{N}}^{-} \equiv P_{\mathcal{N}}$ is given by (II.11), with

$$
\varphi_{i}(q)=\left.\mathrm{e}^{-\mathcal{W}(z)} \tilde{\varphi}_{i}(z)\right|_{z=z(q)}, \quad 1 \leq i \leq \mathcal{N} .
$$

Likewise, the invariance of the space $\mathcal{V}_{\mathcal{N}}$ under the Hamiltonian $H^{-}$is an immediate consequence of the invariance of $\tilde{\mathcal{V}}_{\mathcal{N}}$ under $\tilde{H}$ and Eqs. (II.21) and (II.27). It is important to note that Eq. (II.26) for $P_{\mathcal{N}}$ is compatible with Eq. (II.2). Indeed, from Eqs. (II.18) and (II.22a) it follows that $g$ is proportional to $\dot{z}^{\mathcal{N}}$. Taking

$$
g(z)=\left.\dot{z}(q)^{\mathcal{N}}\right|_{q=q(z)}
$$

and using Eqs. (I.15) and (П.26), it immediately follows that $P_{\mathcal{N}}$ is indeed of the form (II.2). It is also straightforward to check, using Eqs. (II.2), (II.15), (II.19), (II.23), (II.26), and (II.28), that the partner Hamiltonians $H^{ \pm}$are related by (II.10).

At this point we shall briefly summarize the results obtained so far. Given an $\mathcal{N}$ dimensional linear space $\tilde{\mathcal{V}}_{\mathcal{N}}\left(\underline{\text { II.13) }}\right.$ ) and a second-order linear differential operator $\tilde{H} \equiv \tilde{H}^{-}$ (II.14) leaving it invariant, one can construct an $\mathcal{N}$-fold supersymmetric model $\left(\mathbf{H}, Q_{\mathcal{N}}^{ \pm}\right)$ through the following algorithmic steps:

i. Compute the change of variables $z(q)$ using Eq. (II.22a ).

ii. Construct the operator

$$
\tilde{P}_{N}=\dot{z}^{\mathcal{N}}\left(\partial_{z}^{\mathcal{N}}+\sum_{k=0}^{\mathcal{N}-1} \tilde{w}_{k}(z) \partial_{z}^{k}\right)
$$

whose kernel is the linear space $\tilde{\mathcal{V}}_{\mathcal{N}}$.

iii. Compute the operator $\tilde{H}^{+}(\underline{11.16})$ using Eq. (ㅍ.19).

iv. The component Hamiltonians $H^{ \pm}$are obtained from the gauged Hamiltonians $\tilde{H}^{ \pm}$by applying the gauge transformation (II.21), with $\mathcal{W}(z)$ given by (II.22b).

v. Likewise, the operator $P_{\mathcal{N}}$ determining the $\mathcal{N}$-fold supercharges $Q_{\mathcal{N}}^{ \pm}$through Eqs. (II.3)-(II.4) is obtained from $\tilde{P}_{\mathcal{N}}$ via the same gauge transformation, cf. Eq. (II.26).

In practice, the component Hamiltonians $H^{ \pm}$(II.7) are computed using Eq. (II.24) for the potentials $V^{ \pm}(q)$. Note also that the second step in the previous construction is algorithmic, since the operator in parenthesis in Eq. (II.29) can be computed using a formula analogous to (II.12). In most cases, however, the operator $\tilde{P}_{\mathcal{N}}$ is easily found by inspection.

To make the above construction completely symmetric with respect to the partner Hamiltonians $\mathrm{H}^{-}$and $\mathrm{H}^{+}$, and to facilitate comparison of the above results with previous work 
[14, 15], we next introduce two additional differential operators $\bar{P}_{\mathcal{N}}^{+}$and $\bar{H}^{+}$as follows. In the first place, note that from the second intertwining relation in Eq. (II.9) it follows that $\mathrm{H}^{+}$leaves invariant the kernel of the supercharge

$$
P_{\mathcal{N}}^{+}=(-1)^{\mathcal{N}} P_{\mathcal{N}}^{t}=(-1)^{\mathcal{N}} \mathrm{e}^{\mathcal{W}} \tilde{P}_{\mathcal{N}^{t}} \mathrm{e}^{-\mathcal{W}}
$$

Using the identity ${ }^{2}$

$$
\left(\partial_{z}\right)^{t}=\left(\frac{1}{\dot{z}} \partial_{q}\right)^{t}=-\partial_{q} \frac{1}{\dot{z}}=-\dot{z} \partial_{z} \frac{1}{\dot{z}}
$$

and Eq. (II.29) we can write

$$
(-1)^{\mathcal{N}} \tilde{P}_{N}^{t}=\dot{z}^{1-\mathcal{N}} \bar{P}_{\mathcal{N}}^{+} \dot{z}^{\mathcal{N}-1}
$$

where

$$
\bar{P}_{\mathcal{N}}^{+}=\dot{z}^{\mathcal{N}}\left(\partial_{z}^{\mathcal{N}}+\sum_{k=0}^{\mathcal{N}-1}(-1)^{\mathcal{N}-k} \partial_{z}^{k} \tilde{w}_{k}\right)
$$

From Eqs. (II.30) and (II.31) we have

$$
P_{\mathcal{N}}^{+}=\mathrm{e}^{-\mathcal{W}^{+}} \bar{P}_{\mathcal{N}}^{+} \mathrm{e}^{\mathcal{W}^{+}}
$$

where the function $\mathcal{W}^{+}$is given by

$$
\mathcal{W}^{+}=-\mathcal{W}+(\mathcal{N}-1) \ln |\dot{z}|
$$

The invariance of ker $P_{N}^{+}$under $H^{+}$and Eq. ([.33) imply that the operator

$$
\bar{H}^{+}=\mathrm{e}^{\mathcal{W}^{+}} H^{+} \mathrm{e}^{-\mathcal{W}^{+}}
$$

leaves the linear space

$$
\overline{\mathcal{V}}_{\mathcal{N}}^{+}=\operatorname{ker} \bar{P}_{\mathcal{N}}^{+}
$$

invariant. Setting $\mathcal{W}^{-} \equiv \mathcal{W}$ we can express the partner Hamiltonians as

$$
H^{ \pm}=\mathrm{e}^{-\mathcal{W}^{ \pm}} \overline{\tilde{H}}^{ \pm} \mathrm{e}^{\mathcal{W}^{ \pm}}
$$

where the gauged Hamiltonians $\overline{\tilde{H}}^{ \pm}$leave invariant the kernel of the "gauged" supercharges $\overline{\tilde{P}}_{\mathcal{N}}^{ \pm}$respectively given by Eqs. (ㅍ.29) and (ㅍ.32). The "physical" supercharges $P_{\mathcal{N}}^{ \pm}$are related to the gauged supercharges by the equations

$$
P_{\mathcal{N}}^{ \pm}=\mathrm{e}^{-\mathcal{W}} \overline{\tilde{P}}_{\mathcal{N}}^{ \pm} \mathrm{e}^{\mathcal{W}}
$$

In order to express $\mathcal{W}^{ \pm}$in a symmetric way, we introduce the functions

$$
W(q)=\frac{1}{2}\left(\dot{\mathcal{W}}^{-}(q)-\dot{\mathcal{W}}^{+}(q)\right)
$$

\footnotetext{
${ }^{2}$ Note that the transposition has been defined in terms of the variable $q$, cf. Eq. (II.44).
} 
and

$$
E(q)=\frac{\ddot{z}(q)}{\dot{z}(q)} .
$$

From Eq. (II.22a), its immediate consequence

$$
\ddot{z}=A^{\prime},
$$

and Eq. (II.22b) it is straightforward to derive the relation

$$
W=-\frac{Q}{\dot{z}} .
$$

We then have

$$
\mathcal{W}^{ \pm}=\frac{1}{2}(\mathcal{N}-1) \int E(q) \mathrm{d} q \mp \int W(q) \mathrm{d} q=\frac{1}{4}(\mathcal{N}-1) \ln |2 A(z)| \pm \int \frac{Q(z)}{2 A(z)} \mathrm{d} z
$$

cf. [15]. The connection between the gauged Hamiltonians $\bar{H}^{+}$and $\tilde{H}^{+}$follows easily from Eqs. (II.21) and (II.35), namely

$$
\bar{H}^{+}=\mathrm{e}^{-2 \int W(q) \mathrm{d} q} \tilde{H}^{+} \mathrm{e}^{2 \int W(q) \mathrm{d} q} .
$$

Using Eqs. (II.19), (II.22a), (II.25), and (II.41), it is immediate to obtain the following explicit expression for $\bar{H}^{+}$:

$$
-\bar{H}^{+}=A \partial_{z}^{2}-\left(Q+\frac{1}{2}(\mathcal{N}-2) A^{\prime}\right) \partial_{z}+C+(\mathcal{N}-1) Q^{\prime}-A^{\prime} \tilde{w}_{\mathcal{N}-1}-2 A \tilde{w}_{\mathcal{N}-1}^{\prime}
$$

Combining this equation with Eq. (II.14) we obtain the following unified formula for the gauged Hamiltonians $\overline{\tilde{H}}^{ \pm}$:

$$
\begin{aligned}
-\overline{\tilde{H}}^{ \pm}=A \partial_{z}^{2} & -\left( \pm Q+\frac{1}{2}(\mathcal{N}-2) A^{\prime}\right) \partial_{z}+C \\
& +\frac{1}{2}(1 \pm 1)\left((\mathcal{N}-1) Q^{\prime}-A^{\prime} \tilde{w}_{\mathcal{N}-1}-2 A \tilde{w}_{\mathcal{N}-1}^{\prime}\right) .
\end{aligned}
$$

This general formula includes as particular cases the gauged Hamiltonians of the type A and type B models introduced respectively in Refs. [14] and [15]. Indeed, in the type A models we have $\tilde{w}_{\mathcal{N}-1}=0$ and

$$
C=\frac{1}{12}(\mathcal{N}-1)(\mathcal{N}-2) A^{\prime \prime}-\frac{1}{2}(\mathcal{N}-1) Q^{\prime}+R
$$

where $R$ is a constant. Using these relations in Eq. (II.45) we can easily reproduce Eqs. (3.41) and (3.50b), (3.52), (3.55) of Ref. 14] for the gauged Hamiltonians of type A (note that the coordinate $h$ and the function $P$ in the latter reference correspond to our $z$ and $A$, respectively). Likewise, type B models satisfy $\tilde{w}_{\mathcal{N}-1}=-1 / z$ and

$$
C=\frac{1}{12}(\mathcal{N}-1)(\mathcal{N}-2) A^{\prime \prime}-\frac{A^{\prime}}{2 z}-\frac{1}{2}(\mathcal{N}-1) Q^{\prime}-\frac{Q}{\mathcal{N} z}+R,
$$

which immediately lead to Eq. (3.35) of Ref. [15] for the gauged Hamiltonians of type B. 


\section{A NEW FAMILY OF $\mathcal{N}$-FOLD SUPERSYMMETRIC SYSTEMS}

In this section we shall apply the previous results to the construction of a new multiparameter family of $\mathcal{N}$-fold supersymmetric systems for arbitrary $\mathcal{N}$. The key idea in this respect is to choose appropriately the $\mathcal{N}$-dimensional linear space (II.13), in such a way that the linear space of second-order linear differential operators leaving it invariant is nontrivial and can be explicitly computed.

To this end, we shall consider monomial spaces of the form

$$
\tilde{\mathcal{V}}_{\mathcal{N}}=\left\langle z^{\lambda_{1}}, \ldots, z^{\lambda_{\mathcal{N}}}\right\rangle
$$

where the exponents $\lambda_{i}$ are real numbers. All spaces (III.1) left invariant by a nonzero second-order linear differential operator have been classified by Post and Turbiner [16], up to changes of variables and gauge transformations of the form

$$
\psi(z) \mapsto \hat{\psi}(\hat{z})=\hat{z}^{\alpha} \psi\left(\hat{z}^{\beta}\right), \quad \alpha, \beta \in \mathbb{R} .
$$

For $\mathcal{N}>4$ the above classification consists of three equivalence classes, represented by the following "canonical forms":

$$
\begin{aligned}
& \text { A) }\left\langle 1, z, \ldots, z^{\mathcal{N}-1}\right\rangle \\
& \text { B) }\left\langle 1, z, \ldots, z^{\mathcal{N}-2}, z^{\mathcal{N}}\right\rangle \\
& \text { C) }\left\langle 1, z, \ldots, z^{\mathcal{N}_{1}-1}, z^{\lambda}, z^{\lambda+1}, \ldots, z^{\lambda+\mathcal{N}_{2}-1}\right\rangle, \quad \mathcal{N}=\mathcal{N}_{1}+\mathcal{N}_{2} .
\end{aligned}
$$

In the third canonical form $\mathcal{N}_{1}$ and $\mathcal{N}_{2}$ are positive integers, and $\lambda$ is a real number different from $-\mathcal{N}_{2},-\mathcal{N}_{2}+1, \ldots, \mathcal{N}_{1}\left(\lambda \neq-\mathcal{N}_{2}-1, \mathcal{N}_{1}+1\right.$ when $\mathcal{N}_{1}=1$ or $\mathcal{N}_{2}=1$ to prevent the canonical form $\mathrm{C}$ from reducing to $\mathrm{B})$. Due to the freedom in performing changes of variables and gauge transformations (III.2) we can also assume, without loss of generality, that

$$
\lambda>0, \quad \mathcal{N}_{1} \geq \mathcal{N}_{2} .
$$

The $\mathcal{N}$-fold supersymmetric models constructed from the canonical form A following the procedure described in the previous section are nothing but the type A systems introduced in Ref. [13]. Similarly, the models obtained from the second canonical form are the type B systems recently constructed by the authors [15]. In this section we shall therefore derive and completely classify all the $\mathcal{N}$-fold supersymmetric models associated to the last canonical form (III.5), which from now on we shall term type $C$ models for short.

We shall now proceed to the construction of the type $\mathrm{C}$ models by following the algorithmic steps outlined in Section [II. In order to implement this algorithm, we must know at least one nonzero second-order linear differential operator leaving the space (III.5) invariant. Due to the extremely simple nature of the latter space, it is actually straightforward to determine the whole linear space of linear differential operators of order not greater than two preserving it. Indeed, using the techniques described in Ref. [16] it is readily found that the latter space is spanned by the constant multiplication operator 1 and the following operators:

$$
\begin{aligned}
J_{0-} & =\partial_{z}\left(z \partial_{z}-\lambda\right) \\
J_{00} & =z^{2} \partial_{z}^{2} \\
J_{+0} & =z\left(z \partial_{z}-\mathcal{N}_{1}+1\right)\left(z \partial_{z}-\lambda-\mathcal{N}_{2}+1\right) \\
J_{0} & =z \partial_{z} .
\end{aligned}
$$


The most general linear second-order differential operator leaving the space (III.5) invariant is thus given by

$$
-\tilde{H}=a_{1} J_{0-}+a_{2} J_{00}+a_{3} J_{+0}+b_{0} J_{0}+c_{0},
$$

where the coefficients $a_{i}, b_{0}$, and $c_{0}$ are real constants. More explicitly, the coefficients $A(z)$, $B(z)$ and $C(z)$ of $-\tilde{H}$ (cf. (II.14) $)$ are given by

$$
\begin{aligned}
& A(z)=a_{3} z^{3}+a_{2} z^{2}+a_{1} z \\
& B(z)=-(\mathcal{N}+\lambda-3) a_{3} z^{2}+b_{0} z+(1-\lambda) a_{1} \\
& C(z)=\left(\mathcal{N}_{1}-1\right)\left(\mathcal{N}_{2}+\lambda-1\right) a_{3} z+c_{0} .
\end{aligned}
$$

By Eq. (II.25) we have

$$
Q=\frac{1}{2}(\mathcal{N}-2 \lambda) a_{3} z^{2}+b_{1} z+\frac{1}{2}(\mathcal{N}-2 \lambda) a_{1}
$$

with

$$
b_{1}=b_{0}+(\mathcal{N}-2) a_{2} .
$$

From Eqs. (II.22a and (III.9a), the change of variable $z(q)$ is determined in this case by the differential equation

$$
\dot{z}^{2}=2\left(a_{3} z^{3}+a_{2} z^{2}+a_{1} z\right) .
$$

The type $\mathrm{C}$ space (III.5) decomposes in a natural way as the direct sum of two spaces of type A, namely

$$
\left\langle 1, z, \ldots, z^{\mathcal{N}_{1}-1}\right\rangle \oplus z^{\lambda}\left\langle 1, z, \ldots, z^{\mathcal{N}_{2}-1}\right\rangle .
$$

It is important to observe that both subspaces in the latter sum are separately invariant under all of the operators (III.7), and hence under the gauged Hamiltonian $\tilde{H}$. In particular, a result of Turbiner [23] implies that $\tilde{H}$ and $z^{\lambda} \tilde{H} z^{-\lambda}$ are Lie-algebraic operators with respect to the standard realization of the algebra $\mathfrak{s l}(2)$ with generators

$$
J_{-}=\partial_{z}, \quad J_{0}=z \partial_{z}, \quad J_{+}=z^{2} \partial_{z}-n z
$$

and cohomology parameter $n=\mathcal{N}_{1}-1$ and $n=\mathcal{N}_{2}-1$, respectively (this property can also be checked directly using (III.7) ). However, an arbitrary polynomial in the operators (III.14) will not preserve, in general, both type A spaces in (III.13). For this reason, the number of independent first- and second-order operators preserving $\tilde{\mathcal{V}}_{\mathcal{N}}$ is reduced from 8 for type A to 4 for type $\mathrm{C}$. In particular, the set of type $\mathrm{C}$ gauged Hamiltonians does not include all gauged Hamiltonians of type A. In Section $\nabla$ we will discuss this phenomenon from a different viewpoint, namely the breakdown of an underlying symmetry. It should also be noted in this respect that the gauged Hamiltonian of a type B model is not, in general, a polynomial in the $\mathfrak{s l}(2)$ generators (III.14).

The operators $J_{0-}, J_{00}$, and $J_{0}$ obviously leave invariant the spaces $\left\langle 1, z, \ldots, z^{\mathcal{N}_{1}-1}\right\rangle$ and $z^{\lambda}\left\langle 1, z, \ldots, z^{\mathcal{N}_{2}-1}\right\rangle$ for arbitrary positive-integer values of $\mathcal{N}_{1}$ and $\mathcal{N}_{2}$. It follows from Eq. (III.8) that the gauged Hamiltonian $\tilde{H}$ will also leave invariant the latter spaces for all $\mathcal{N}_{1}, \mathcal{N}_{2} \in \mathbb{N}$ provided that the coefficient $a_{3}$ vanishes. When this is the case, Eq. (II.37) implies that the Hamiltonian $H^{-}$preserves the two infinite ascending sequences of spaces

$$
\mathcal{V}_{\mathcal{N}_{i}}^{-} \equiv \mathrm{e}^{-\mathcal{W}^{-}(q)} z(q)^{(i-1) \lambda}\left\langle 1, z(q), \ldots, z(q)^{\mathcal{N}_{i}-1}\right\rangle ; \quad i=1,2, \quad \mathcal{N}_{i} \in \mathbb{N}
$$


Hence for $a_{3}=0$ the type $\mathrm{C}$ component Hamiltonian $H^{-}$is solvable in Turbiner's sense [24, 25] (see also [12, 17]). We will see shortly that in this case the other Hamiltonian $H^{+}$ is simultaneously solvable, as for type A models.

The $\mathcal{N}$ th-order linear differential operator $\tilde{P}_{\mathcal{N}}$ of the form (II.29) having as kernel the type $\mathrm{C}$ space (III.5) is easily found to be

$$
\tilde{P}_{\mathcal{N}}=\dot{z}^{\mathcal{N}}\left(\partial_{z}+\frac{\mathcal{N}_{1}-\lambda}{z}\right)^{\mathcal{N}_{2}} \partial_{z}^{\mathcal{N}_{1}}
$$

In particular, the function $\tilde{w}_{\mathcal{N}-1}$ is given in this case by

$$
\tilde{w}_{\mathcal{N}-1}=\frac{\mathcal{N}_{2}\left(\mathcal{N}_{1}-\lambda\right)}{z}
$$

To compute the operator $P_{\mathcal{N}}$ determining the supercharges $Q_{\mathcal{N}}^{ \pm}$, let us introduce the function

$$
F(q)=\frac{\dot{z}(q)}{z(q)}
$$

related to $E$ by the identity (see Eq. (II.40)

$$
\dot{F}=E F-F^{2} \text {. }
$$

Making repeated use of the equality

$$
\left(\partial_{z}+\frac{\alpha}{z}\right) \dot{z}^{-k}=\dot{z}^{-1}\left(\partial_{q}+\alpha F\right) \dot{z}^{-k}=\dot{z}^{-k-1}\left(\partial_{q}+\alpha F-k E\right)
$$

we immediately obtain

$$
\tilde{P}_{\mathcal{N}}=\prod_{i=\mathcal{N}_{1}}^{\mathcal{N}-1}\left(\partial_{q}+\left(\mathcal{N}_{1}-\lambda\right) F-i E\right) \cdot \prod_{i=0}^{\mathcal{N}_{1}-1}\left(\partial_{q}-i E\right),
$$

where the products of operators are ordered according to the following definition:

$$
\prod_{i=i_{0}}^{i_{1}} A_{i} \equiv A_{i_{1}} A_{i_{1}-1} \cdots A_{i_{0}}
$$

Using the identity

$$
\mathrm{e}^{-\mathcal{W}} \partial_{q} \mathrm{e}^{\mathcal{W}}=\partial_{q}+\dot{\mathcal{W}}=\partial_{q}+\frac{1}{2}(\mathcal{N}-1) E(q)+W(q)
$$

and Eq. (I.26) we finally obtain the following explicit formula for the operator $P_{\mathcal{N}}$ of type C:

$$
\begin{aligned}
P_{\mathcal{N}}=\prod_{i=\mathcal{N}_{1}}^{\mathcal{N}-1}\left(\partial_{q}+W+\left(\mathcal{N}_{1}-\lambda\right) F+\frac{1}{2}(\mathcal{N}\right. & -1-2 i) E) \\
& \times \prod_{i=0}^{\mathcal{N}_{1}-1}\left(\partial_{q}+W+\frac{1}{2}(\mathcal{N}-1-2 i) E\right)
\end{aligned}
$$


It is clear from the previous expression that the supercharge (III.22) reduces to its type $\mathrm{A}$ counterpart for $\lambda=\mathcal{N}_{1}=\mathcal{N}-1$, and to the type $\mathrm{B}$ one for $\lambda=\mathcal{N}_{1}+1=\mathcal{N}$. We can thus formally regard the type $\mathrm{C}$ supercharge as a deformation of those of types $\mathrm{A}$ and $\mathrm{B}$ depending on the two parameters $\mathcal{N}_{1}$ and $\lambda$.

The pair of type $\mathrm{C}$ potentials $V^{ \pm}$can be expressed in terms of the functions $E, F$ and $W$ using the following identities, which are easily derived from Eqs. (II.22a), (II.40), (II.41), (III.16), and (III.17):

$$
\begin{aligned}
& A^{\prime} \tilde{w}_{\mathcal{N}-1}=\mathcal{N}_{2}\left(\mathcal{N}_{1}-\lambda\right) E F, \quad 2 A \tilde{w}_{\mathcal{N}-1}^{\prime}=-\mathcal{N}_{2}\left(\mathcal{N}_{1}-\lambda\right) F^{2}, \\
& 2 A=z^{2} F^{2}, \quad \frac{A^{\prime 2}}{2 A}=E^{2}, \quad A^{\prime \prime}=\dot{E}+E^{2}, \\
& \frac{Q^{2}}{2 A}=W^{2}, \quad \frac{Q A^{\prime}}{2 A}=-E W, \quad Q^{\prime}=-(\dot{W}+E W) .
\end{aligned}
$$

Substituting the above formulas into Eq. (II.24) and using the relation (III.18), we finally obtain $^{3}$

$H^{ \pm}=-\frac{1}{2} \partial_{q}^{2}+\frac{W^{2}}{2}-\frac{\mathcal{N}_{2}\left(\mathcal{N}_{1}-\lambda\right)}{4} F^{2}-\frac{\mathcal{N}^{2}-1}{24}\left(2 \dot{E}-E^{2}\right) \pm\left[\frac{\mathcal{N}}{2} \dot{W}+\frac{\mathcal{N}_{2}\left(\mathcal{N}_{1}-\lambda\right)}{2} \dot{F}\right]-R$,

where $R$ is a constant.

From Eqs. (II.32) and (III.15) it follows that the operator $\bar{P}_{\mathcal{N}}^{+}$is given in this case by

$$
\bar{P}_{\mathcal{N}}^{+}=\dot{z}^{\mathcal{N}} \partial_{z}^{\mathcal{N}_{1}}\left(\partial_{z}-\frac{\mathcal{N}_{1}-\lambda}{z}\right)^{\mathcal{N}_{2}}
$$

Its kernel $\overline{\mathcal{V}}_{\mathcal{N}}^{+}$is easily computed (cf. Eq. ([1.36) $)$, with the result

$$
\overline{\mathcal{V}}_{\mathcal{N}}^{+}=z^{\mathcal{N}_{2}}\left\langle 1, z, \ldots, z^{\mathcal{N}_{1}-1}, z^{\bar{\lambda}}, z^{\bar{\lambda}+1}, \ldots, z^{\bar{\lambda}+\mathcal{N}_{2}-1}\right\rangle
$$

where

$$
\bar{\lambda}=\mathcal{N}_{1}-\mathcal{N}_{2}-\lambda \text {. }
$$

The space $\overline{\mathcal{V}}_{\mathcal{N}}^{+}$can obviously be transformed into the type $\mathrm{C}$ canonical form (III.5) by the gauge transformation $\psi(z) \mapsto \hat{\psi}(z)=z^{-\mathcal{N}_{2}} \psi(z)$. Since $\bar{\lambda}$ is negative if $\lambda>\mathcal{N}_{1}-\mathcal{N}_{2}$, strictly speaking it is necessary in this case to perform an additional gauge transformation and change of variable (III.2) to take the space (III.26) into the canonical form (III.5)-(III.6) It is preferable, however, not to enforce the first restriction $\lambda>0$ in Eq. (III.6) in the sequel (cf. the discussion following Eq. (III.41)), the positivity of $\lambda$ being immaterial in other respects. Hence from now on we shall only impose on $\lambda$ the restriction needed to ensure that the type $\mathrm{C}$ space (III.5) does not reduce to the type A or B canonical forms, namely

$$
\lambda \in \mathbb{R} \backslash\left\{-\mathcal{N}_{2},-\mathcal{N}_{2}+1, \ldots, \mathcal{N}_{1}\right\},
$$

with $\lambda \neq-\mathcal{N}_{2}-1, \mathcal{N}_{1}+1$ if $\mathcal{N}_{1}=1$ or $\mathcal{N}_{2}=1$. It is easily seen from Eq. (III.27) that $\bar{\lambda}$ is restricted in exactly the same way as $\lambda$.

\footnotetext{
${ }^{3}$ Due to the relation (III.18), this expression is not unique.
} 
From Eq. (II.26) it follows that the gauged Hamiltonians $\bar{H}^{+}\left[a_{i}, b_{1}, c_{0}, \lambda\right]$ and $\tilde{H}^{-}\left[\bar{a}_{i}, \bar{b}_{1}, \bar{c}_{0}, \bar{\lambda}\right]$ are related by the gauge transformation

$$
\bar{H}^{+}\left[a_{i}, b_{1}, c_{0}, \lambda\right]=z^{\mathcal{N}_{2}} \tilde{H}^{-}\left[\bar{a}_{i}, \bar{b}_{1}, \bar{c}_{0}, \bar{\lambda}\right] z^{-\mathcal{N}_{2}}
$$

for a suitable choice of the parameters $\bar{a}_{i}, \bar{b}_{1}$, and $\bar{c}_{0}$. The latter equality implies, by the uniqueness (up to an additive constant) of the physical Hamiltonian associated to a given gauged Hamiltonian, the important relation

$$
H^{+}\left[a_{i}, b_{1}, c_{0}, \lambda\right]=H^{-}\left[\bar{a}_{i}, \bar{b}_{1}, \bar{c}_{0}, \bar{\lambda}\right] .
$$

In other words, the type $\mathrm{C} \mathcal{N}$-fold supersymmetric models we shall obtain are guaranteed to be (formally) shape invariant [26]. Explicitly, the parameters $\left(\bar{a}_{i}, \bar{b}_{1}, \bar{c}_{0}\right)$ are given by,

$$
\begin{aligned}
& \bar{a}_{i}=a_{i}, \quad i=1,2,3, \\
& \bar{b}_{1}=-b_{1}+2 \mathcal{N}_{2} a_{2}, \\
& \bar{c}_{0}=c_{0}+\left(\mathcal{N}_{1}-1\right)\left(b_{1}-\mathcal{N}_{2} a_{2}\right) .
\end{aligned}
$$

Since $a_{3}=0$ implies $\bar{a}_{3}=0$ and vice versa, it follows that $H^{-}$and $H^{+}$are always simultaneously solvable.

We shall next examine the explicit forms of the subspaces $\mathcal{V}_{\mathcal{N}}^{ \pm}$preserved by the type $\mathrm{C}$ Hamiltonians $H^{ \pm}$. We first note from Eqs. (III.9a) and (III.10) that the second term of the last expression for $\mathcal{W}^{ \pm}$in Eq. (II.42) is given by

$$
\int \frac{Q(z)}{2 A(z)} \mathrm{d} z=\frac{\mathcal{N}-2 \lambda}{4} \ln |z|+\left(\frac{b_{1}}{2}-\frac{\mathcal{N}-2 \lambda}{4} a_{2}\right) \int \frac{z}{A(z)} \mathrm{d} z .
$$

Introducing the new parameters

$$
\alpha^{-}=\frac{1}{2}-\lambda, \quad \alpha^{+}=\mathcal{N}_{2}-\mathcal{N}_{1}+\lambda+\frac{1}{2}=\frac{1}{2}-\bar{\lambda}
$$

we obtain the following expression for the gauge factors of the type $\mathrm{C}$ models:

$$
\begin{aligned}
\mathcal{W}^{ \pm}=-\left(\frac{\alpha^{ \pm}}{2}-\frac{1 \pm 1}{2} \mathcal{N}_{2}\right) \ln |z|+\frac{\mathcal{N}-1}{4} \ln \mid & \left|\frac{A(z)}{z}\right| \\
& \pm\left(\frac{b_{1}}{2}-\frac{\mathcal{N}-2 \lambda}{4} a_{2}\right) \int \frac{z}{A(z)} \mathrm{d} z
\end{aligned}
$$

where an irrelevant constant term has been dropped. By Eq. (II.37), the subspaces $\mathcal{V}_{\mathcal{N}}^{ \pm}$ invariant under the Hamiltonians $H^{ \pm}$, which provide the algebraically computable wave functions (not taking their normalizability into account), are obviously given by

$$
\mathcal{V}_{\mathcal{N}}^{ \pm}=\mathrm{e}^{-\mathcal{W}_{ \pm}} \overline{\tilde{\mathcal{V}}}_{\mathcal{N}}^{ \pm}
$$

From Eqs. (III.5), (III.26) and (III.34) we finally have

$$
\begin{aligned}
\mathcal{V}_{\mathcal{N}}^{ \pm} & =\left.\mathrm{e}^{-\mathcal{U}^{ \pm}} z^{\frac{1}{2} \alpha^{ \pm}}\left\langle 1, z, \ldots, z^{\mathcal{N}_{1}-1}\right\rangle \oplus \mathrm{e}^{-\mathcal{U}^{ \pm}} z^{\frac{1}{2}\left(1-\alpha^{ \pm}\right)}\left\langle 1, z, \ldots, z^{\mathcal{N}_{2}-1}\right\rangle\right|_{z=z(q)} \\
& \equiv \mathcal{V}_{\mathcal{N}_{1}}^{ \pm} \oplus \mathcal{V}_{\mathcal{N}_{2}}^{ \pm}
\end{aligned}
$$


where the new gauge factors $\mathrm{e}^{-\mathcal{U}^{ \pm}}$are defined by

$$
\mathrm{e}^{-\mathcal{U}^{ \pm}}=\left|\frac{A(z)}{z}\right|^{-\frac{1}{4}(\mathcal{N}-1)} \exp \left[\mp\left(\frac{b_{1}}{2}-\frac{\mathcal{N}-2 \lambda}{4} a_{2}\right) \int \frac{z}{A(z)} \mathrm{d} z\right] \text {. }
$$

The last step in our construction is the computation of the component Hamiltonians $H^{ \pm}$ using Eqs. (III.7) and (II.24). This is, technically speaking, the most delicate step, since it involves the explicit evaluation of the elliptic integral

$$
\int\left[2\left(a_{3} z^{3}+a_{2} z^{2}+a_{1} z\right)\right]^{-1 / 2} \mathrm{~d} z= \pm\left(q-q_{0}\right)
$$

needed to compute the change of variable $z=z(q)$, cf. Eq. (III.12).

The value of the integral (III.38), and hence the corresponding change of variable, depends on the position of the roots of the polynomial $A(z)$ in the complex plane. We should therefore classify the polynomial $A(z)$ into (real) canonical forms according to the position of its roots, using changes of variables and gauge transformations

$$
\psi(z) \mapsto \hat{\psi}(\hat{z})=\left.\mu(z) \psi(z)\right|_{z=\zeta(\hat{z})}
$$

that preserve the form of the type $\mathrm{C}$ space (III.5). This task is hindered by the fact that, contrary to what happens in the analogous classification of the one-dimensional Lie-algebraic and type A $\mathcal{N}$-fold supersymmetric Hamiltonians [14, 19, 22], the space (III.5) is not invariant under translations $z=\hat{z}+z_{0}$. Fortunately, however, this space is invariant under dilations

$$
\psi(z) \mapsto \hat{\psi}(\hat{z}) \equiv \psi(\alpha \hat{z}), \quad \alpha \in \mathbb{R},
$$

and is form-invariant under special projective transformations

$$
\psi(z) \mapsto \hat{\psi}(\hat{z}) \equiv \hat{z}^{s} \psi\left(\hat{z}^{-1}\right), \quad s=\mathcal{N}_{1}-1, \mathcal{N}_{2}+\lambda-1
$$

Since, however, neither projective transformation (III.41) preserves both conditions (III.6), we must drop one of these conditions if we insist on using projective transformations to bring $A(z)$ into canonical form. From now on we shall assume that only the second condition $\mathcal{N}_{1} \geq N_{2}$ in Eq. (III.6) holds, which entails the choice $s=\mathcal{N}_{1}-1$ in Eq. (III.41).

The transformations (III.40)-(III.41) map the gauged Hamiltonian $\tilde{H}$ (II.14) into the operator $\hat{H}$ respectively given by

$$
-\hat{H}=-\left.\tilde{H}\right|_{z=\alpha \hat{z}}=\frac{1}{\alpha^{2}} A(\alpha \hat{z}) \frac{\mathrm{d}^{2}}{\mathrm{~d} \hat{z}^{2}}+\frac{1}{\alpha} B(\alpha \hat{z}) \frac{\mathrm{d}}{\mathrm{d} \hat{z}}+C(\alpha \hat{z})
$$

and

$$
-\hat{H}=-\left.\hat{z}^{s} \tilde{H} \hat{z}^{-s}\right|_{z=\hat{z}^{-1}}=A\left(\hat{z}^{-1}\right)\left(-\hat{z}^{2} \frac{\mathrm{d}}{\mathrm{d} \hat{z}}+s \hat{z}\right)^{2}+B\left(\hat{z}^{-1}\right)\left(-\hat{z}^{2} \frac{\mathrm{d}}{\mathrm{d} \hat{z}}+s \hat{z}\right)+C\left(\hat{z}^{-1}\right) .
$$

In particular, the polynomial $A(z)$ transforms under dilations and special projective transformations respectively as

and

$$
A(z) \mapsto \hat{A}(\hat{z}) \equiv \frac{1}{\alpha^{2}} A(\alpha \hat{z})
$$

$$
A(z) \mapsto \hat{A}(\hat{z}) \equiv \hat{z}^{4} A\left(\hat{z}^{-1}\right) .
$$

With the help of the transformations (III.42)-(III.43) it is readily shown that $A(z)$ can be cast into one of the canonical forms listed in Table I. The discussion following Eq. (III.12) implies that the type $\mathrm{C}$ models corresponding to the first two canonical forms in Table \. or to the third one with $m=0$, are not only quasi-solvable but also solvable. 
TABLE I: Canonical forms for the polynomial $A(z)$ (III.9a). In this Table $\nu>0,0 \leq m \leq 1$ and $m^{\prime}=1-m(m \neq 1$ in Case 3 and $m \neq 0,1$ in Case 4 to avoid duplications).

\begin{tabular}{l}
\hline \hline 1) $2 z$ \\
2) $\pm \frac{1}{2} \nu z^{2}$ \\
3) $\pm 2 \nu z(1-z)(1-m z)$ \\
4) $2 \nu z(1-z)\left(m^{\prime}+m z\right)$ \\
5) $\frac{1}{2} \nu z\left(z^{2}+2(1-2 m) z+1\right)$ \\
\hline \hline
\end{tabular}

\section{CLASSIFICATION OF THE TYPE C MODELS}

We shall now explicitly compute the type $\mathrm{C}$ models associated to each of the canonical forms in Table I. Note that, by Eq. (III.12), a rescaling of the coefficients $a_{i}, b_{1}, c_{0}$ by an overall nonzero constant factor $\nu$ has the following effect on the change of variable $z(q)$ :

$$
z\left(q ; \nu a_{i}, \nu b_{1}, \nu c_{0}\right)=z\left(\sqrt{\nu} q ; a_{i}, b_{1}, c_{0}\right) .
$$

From this equation and Eqs. (II.24), (II.25), (II.40)-(II.42), and (III.17) we easily obtain the identities

$$
\begin{aligned}
E\left(q ; \nu a_{i}, \nu b_{1}, \nu c_{0}\right) & =\sqrt{\nu} E\left(\sqrt{\nu} q ; a_{i}, b_{1}, c_{0}\right) \\
F\left(q ; \nu a_{i}, \nu b_{1}, \nu c_{0}\right) & =\sqrt{\nu} F\left(\sqrt{\nu} q ; a_{i}, b_{1}, c_{0}\right) \\
W\left(q ; \nu a_{i}, \nu b_{1}, \nu c_{0}\right) & =\sqrt{\nu} W\left(\sqrt{\nu} q ; a_{i}, b_{1}, c_{0}\right) \\
\mathcal{W}^{ \pm}\left(q ; \nu a_{i}, \nu b_{1}, \nu c_{0}\right) & =\mathcal{W}^{ \pm}\left(\sqrt{\nu} q ; a_{i}, b_{1}, c_{0}\right) \\
V^{ \pm}\left(q ; \nu a_{i}, \nu b_{1}, \nu c_{0}\right) & =\nu V^{ \pm}\left(\sqrt{\nu} q ; a_{i}, b_{1}, c_{0}\right) .
\end{aligned}
$$

We shall therefore set $\nu=1$ in the canonical forms 2)-5), the models corresponding to an arbitrary value of $\nu$ following easily from Eqs. (IV.1) and (IV.2). It should also be obvious from Eq. (III.38) that the change of variable $z(q)$, and hence the functions $E, F, W$ and the potentials $V^{ \pm}$determining each model, are defined up to the transformation $q \mapsto \pm\left(q-q_{0}\right)$, where $q_{0} \in \mathbb{R}$ is a constant. We shall make use of this observation to simplify the expressions for $E, F, W$, and $V^{ \pm}$.

Case 1. $A(z)=2 z$.

Change of variable: $z=q^{2}$.

Supercharge:

$$
E=\frac{1}{q}, \quad F=\frac{2}{q}, \quad W=-\frac{b_{1}}{2} q+\frac{1-\mathcal{N}-2 \alpha^{-}}{2 q}
$$

Potentials:

$$
V^{ \pm}=\frac{1}{8} b_{1}^{2} q^{2}+\frac{\alpha^{ \pm}\left(\alpha^{ \pm}-1\right)}{2 q^{2}} \mp \frac{\mathcal{N}}{4} b_{1}+V_{0} .
$$

Here, and in what follows, $V_{0}$ denotes an arbitrary constant. 
Solvable sectors:

$$
\begin{aligned}
& \mathcal{V}_{\mathcal{N}_{1}}^{ \pm}=\mathrm{e}^{\mp \frac{1}{4} b_{1} q^{2}} q^{\alpha^{ \pm}}\left\langle 1, q^{2}, \ldots, q^{2\left(\mathcal{N}_{1}-1\right)}\right\rangle, \\
& \mathcal{V}_{\mathcal{N}_{2}}^{ \pm}=\mathrm{e}^{\mp \frac{1}{4} b_{1} q^{2}} q^{1-\alpha^{ \pm}}\left\langle 1, q^{2}, \ldots, q^{2\left(\mathcal{N}_{2}-1\right)}\right\rangle,
\end{aligned}
$$

where the linear spaces $\mathcal{V}_{\mathcal{N}_{i}}^{ \pm}$are defined in Eq. (III.36). This case corresponds to a solvable model. The potentials (IV.4 are singular at the origin, and thus their Hamiltonians may be naturally defined on, e.g., the half-line $S=(0, \infty)$. In this case, we see from Eqs. (IV.5) that the normalizability of the solvable sectors depends on the value of $\alpha^{ \pm}$(or $\lambda$ ) and the sign of $b_{1}$. The finiteness of the $L^{2}$ norm in the solvable sectors yields the following conditions: ${ }^{4}$

$$
\begin{array}{lll}
\mathcal{V}_{\mathcal{N}_{1}}^{+} \subset L^{2}(S) & \Longleftrightarrow b_{1}>0, & \lambda>\mathcal{N}_{1}-\mathcal{N}_{2}-1, \\
\mathcal{V}_{\mathcal{N}_{2}}^{+} \subset L^{2}(S) & \Longleftrightarrow \quad b_{1}>0, \quad \lambda<\mathcal{N}_{1}-\mathcal{N}_{2}+1, \\
\mathcal{V}_{\mathcal{N}_{1}}^{-} \subset L^{2}(S) & \Longleftrightarrow \quad b_{1}<0, \quad \lambda<1, \\
\mathcal{V}_{\mathcal{N}_{2}}^{-} \subset L^{2}(S) & \Longleftrightarrow \quad b_{1}<0, \quad \lambda>-1 .
\end{array}
$$

Case 2a. $A(z)=z^{2} / 2$.

Change of variable: $z=\mathrm{e}^{q}$.

Supercharge:

$$
E=F=1, \quad W=-b_{1}
$$

Potentials:

$$
V^{ \pm}=V_{0}
$$

Thus both potentials are equal and trivial in this case.

Case 2b. $A(z)=-z^{2} / 2$.

The formulas for the supercharge and the potentials for this case can be easily deduced from those of the preceding one by applying Eqs. (IV.2) with $\nu=-1$.

Case 3a. $A(z)=2 z(1-z)(1-m z)$.

Change of variable: $z=\operatorname{sn}^{2} q$.

Here, and in the following cases, the Jacobian elliptic functions have modulus $k=\sqrt{m}$. Strictly speaking, if $0<m<1$ the above change of variable is only valid in one of the two regions in which $A(z)$ is positive, namely the interval $0<z<1$. In the second region of positivity $1 / m<z$ the change of variable is $z=1 /\left(m \mathrm{sn}^{2} q\right)$. However, since the projective transformation $w=1 /(m z)$ leaves $A$ invariant and maps the interval $(0,1)$ into the half-line $(1 / m, \infty)$, we need not consider the second change of variable.

Supercharge:

$$
\begin{gathered}
E=\frac{3 m \operatorname{sn}^{4} q-2(1+m) \operatorname{sn}^{2} q+1}{\operatorname{sn} q \operatorname{cn} q \operatorname{dn} q}, \quad F=2 \frac{\operatorname{cn} q \operatorname{dn} q}{\operatorname{sn} q} \\
W=\frac{\left(1-\mathcal{N}-2 \alpha^{-}\right)\left(1+m \operatorname{sn}^{4} q\right)-b_{1} \operatorname{sn}^{2} q}{2 \operatorname{sn} q \operatorname{cn} q \operatorname{dn} q}
\end{gathered}
$$

\footnotetext{
${ }^{4}$ For the solvable sectors to be included in a Hilbert space on which the Hamiltonians $H^{ \pm}$are self-adjoint, we need of course to impose a stronger restriction on the parameters coming from the boundary condition at the endpoint $q=0$, which is usually taken as $\lim _{q \rightarrow 0+} q^{-1 / 2} \psi(q)=0$. See also the discussion in Section VI
} 
Potentials:

$$
\begin{aligned}
V^{ \pm}=\frac{m}{2} \alpha^{\mp}\left(\alpha^{\mp}-1\right) \operatorname{sn}^{2} q+\frac{\alpha^{ \pm}\left(\alpha^{ \pm}-1\right)}{2 \operatorname{sn}^{2} q}+ & \frac{m^{\prime}}{2} \frac{\beta^{ \pm}\left(\beta^{ \pm}-1\right)}{\operatorname{cn}^{2} q} \\
& -\frac{m^{\prime}}{2} \frac{\beta^{\mp}\left(\beta^{\mp}-1\right)}{\operatorname{dn}^{2} q}+\frac{m^{\prime} \mathcal{N}}{2} \beta^{ \pm}+V_{0} .
\end{aligned}
$$

Parameters:

$$
\beta^{ \pm}=\frac{1}{2}(1-\mathcal{N}) \pm \frac{1}{2 m^{\prime}}\left(b_{1}+(1+m)\left(\mathcal{N}+2 \alpha^{-}-1\right)\right) .
$$

Solvable sectors:

$$
\begin{aligned}
& \mathcal{V}_{\mathcal{N}_{1}}^{ \pm}=(\operatorname{sn} q)^{\alpha^{ \pm}}(\operatorname{cn} q)^{\beta^{ \pm}}(\operatorname{dn} q)^{\beta^{\mp}}\left\langle 1, \operatorname{sn}^{2} q, \ldots,(\operatorname{sn} q)^{2\left(\mathcal{N}_{1}-1\right)}\right\rangle \\
& \mathcal{V}_{\mathcal{N}_{2}}^{ \pm}=(\operatorname{sn} q)^{1-\alpha^{ \pm}}(\operatorname{cn} q)^{\beta^{ \pm}}(\operatorname{dn} q)^{\beta^{\mp}}\left\langle 1, \operatorname{sn}^{2} q, \ldots,(\operatorname{sn} q)^{2\left(\mathcal{N}_{2}-1\right)}\right\rangle .
\end{aligned}
$$

It is worth mentioning that in this case the potentials $V^{+}$and $V^{-}$are related by a complex translation (up to a constant term), namely

$$
V^{+}(q)=V^{-}\left(q+\mathrm{i} K^{\prime}\right)+\frac{\mathcal{N} m^{\prime}}{2}\left(\beta^{-}-\beta^{+}\right)
$$

In the latter equation $K^{\prime} \equiv K\left(m^{\prime}\right)$ is the complete elliptic integral of the first kind, defined by

$$
K(m) \equiv \int_{0}^{\pi / 2} \frac{d t}{\sqrt{1-m \sin ^{2} t}} .
$$

In general, the potentials (IV.10) have real singularities at the points $q=n K(n \in \mathbb{Z})$, and thus their Hamiltonians may be naturally defined on, e.g., $S=(0, K)$. In this case, we see from Eqs. (IV.12) that the normalizability of the solvable sectors depends on the value of $\alpha^{ \pm}$and $\beta^{ \pm}$. The restrictions on the parameters coming from the finiteness of the $L^{2}$ norm in the solvable sectors are now given by

$$
\begin{array}{ll}
\mathcal{V}_{\mathcal{N}_{1}}^{ \pm} \subset L^{2}(S) & \Longleftrightarrow \alpha^{ \pm}>-\frac{1}{2}, \quad \beta^{ \pm}>-\frac{1}{2}, \\
\mathcal{V}_{\mathcal{N}_{2}}^{ \pm} \subset L^{2}(S) & \Longleftrightarrow \alpha^{ \pm}<\frac{3}{2}, \quad \beta^{ \pm}>-\frac{1}{2} .
\end{array}
$$

As previously discussed, when $m=0$ the polynomial $A(z)$ is of second degree, and hence the Hamiltonians $H^{ \pm}$are solvable. The formulas for the supercharge, the potentials and the solvable sectors are obtained from Eqs. (IV.9)-(IV.12) by setting $m=0, m^{\prime}=1$, and $(\operatorname{sn} q, \operatorname{cn} q, \operatorname{dn} q)=(\sin q, \cos q, 1)$.

Case 3b. $A(z)=-2 z(1-z)(1-m z)$.

As in Case $2 \mathrm{~b}$, the formulas for this case can follow from those of the preceding one using Eqs. (IV.2) with $\nu=-1$. The following well-known identities [27] may be of help in this case:

$$
\operatorname{sn}(\mathrm{i} q ; m)=\mathrm{i} \frac{\operatorname{sn}\left(q ; m^{\prime}\right)}{\operatorname{cn}\left(q ; m^{\prime}\right)}, \quad \operatorname{cn}(\mathrm{i} q ; m)=\frac{1}{\operatorname{cn}\left(q ; m^{\prime}\right)}, \quad \operatorname{dn}(\mathrm{i} q ; m)=\frac{\operatorname{dn}\left(q ; m^{\prime}\right)}{\operatorname{cn}\left(q ; m^{\prime}\right)}
$$


The value $m=0$ yields again solvable models, whose supercharge, potentials and solvable sectors follow from Eqs. (IV.9) - (IV.12) by setting $m=0, m^{\prime}=1$, and ( $\operatorname{sn} \mathrm{i} q$, cn i $q$, dni $q$ ) = (i $\sinh q, \cosh q, 1)$. This case deserves further discussion, since the resulting potentials

$$
V^{ \pm}=\frac{\alpha^{ \pm}\left(\alpha^{ \pm}-1\right)}{2 \sinh ^{2} q}-\frac{\beta^{ \pm}\left(\beta^{ \pm}-1\right)}{2 \cosh ^{2} q}+\frac{1}{2} \beta^{\mp}\left(\beta^{\mp}-1\right)-\frac{\mathcal{N}}{2} \beta^{ \pm}-V_{0},
$$

are now of hyperbolic type, and hence are not periodic on $\mathbb{R}$. The solvable sectors are given by

$$
\begin{aligned}
& \mathcal{V}_{\mathcal{N}_{1}}^{ \pm}=(\sinh q)^{\alpha^{ \pm}}(\cosh q)^{\beta^{ \pm}}\left\langle 1, \sinh ^{2} q, \ldots,(\sinh q)^{2\left(\mathcal{N}_{1}-1\right)}\right\rangle \\
& \mathcal{V}_{\mathcal{N}_{2}}^{ \pm}=(\sinh q)^{1-\alpha^{ \pm}}(\cosh q)^{\beta^{ \pm}}\left\langle 1, \sinh ^{2} q, \ldots,(\sinh q)^{2\left(\mathcal{N}_{2}-1\right)}\right\rangle .
\end{aligned}
$$

The potentials (IV.16) are singular only at the origin, and thus their Hamiltonians may be naturally defined on, e.g., $S=(0, \infty)$. The finiteness of the $L^{2}$ norm in the solvable sectors leads to the following restrictions:

$$
\begin{aligned}
& \mathcal{V}_{\mathcal{N}_{1}}^{ \pm} \subset L^{2}(S) \quad \Longleftrightarrow \quad-\frac{1}{2}<\alpha^{ \pm}<-\beta^{ \pm}-2 \mathcal{N}_{1}+2, \\
& \mathcal{V}_{\mathcal{N}_{2}}^{ \pm} \subset L^{2}(S) \quad \Longleftrightarrow \quad \beta^{ \pm}+2 \mathcal{N}_{2}-1<\alpha^{ \pm}<\frac{3}{2} .
\end{aligned}
$$

Note that the above inequalities cannot be satisfied unless $\beta^{ \pm}<-2 \mathcal{N}_{i}+5 / 2, i=1,2$.

Case 4. $A(z)=2 z(1-z)\left(m^{\prime}+m z\right)$.

Change of variable: $z=\mathrm{cn}^{2} q$.

Again, the above change of variable is only valid in the interval $0<z<1$. In the second region of positivity of $A(z)$, namely the half-line $z<-m^{\prime} / m$, the correct change of variable is $z=-m^{\prime} /\left(m \mathrm{cn}^{2} q\right)$. As before, we shall restrict ourselves to the interval $0<z<1$, since the projective transformation $w=-m^{\prime} /(m z)$ maps this interval into the half-line $\left(-\infty,-m^{\prime} / m\right)$ and leaves $A$ invariant.

Supercharge:

$$
\begin{gathered}
E=\frac{3 m \operatorname{sn}^{4} q-2(1+m) \operatorname{sn}^{2} q+1}{\operatorname{sn} q \operatorname{cn} q \operatorname{dn} q}, \quad F=-2 \frac{\operatorname{sn} q \operatorname{dn} q}{\operatorname{cn} q}, \\
W=\frac{\left(1-\mathcal{N}-2 \alpha^{-}\right)\left(m \operatorname{cn}^{4} q-m^{\prime}\right)+b_{1} \operatorname{cn}^{2} q}{2 \operatorname{sn} q \operatorname{cn} q \operatorname{dn} q}
\end{gathered}
$$

Potentials:

$$
\begin{aligned}
V^{ \pm}=\frac{m}{2} \alpha^{\mp}\left(\alpha^{\mp}-1\right) \operatorname{sn}^{2} q+ & \frac{\beta^{ \pm}\left(\beta^{ \pm}-1\right)}{2 \operatorname{sn}^{2} q}+\frac{m^{\prime}}{2} \frac{\alpha^{ \pm}\left(\alpha^{ \pm}-1\right)}{\operatorname{cn}^{2} q} \\
& -\frac{m^{\prime}}{2} \frac{\beta^{\mp}\left(\beta^{\mp}-1\right)}{\operatorname{dn}^{2} q}+\frac{m}{2}\left(\mathcal{N}_{2}-\mathcal{N}_{1}\right) \alpha^{ \pm}+\frac{\mathcal{N}}{2} \beta^{ \pm}+V_{0} .
\end{aligned}
$$

Parameters:

$$
\beta^{ \pm}=\frac{1}{2}(1-\mathcal{N}) \pm \frac{1}{2}\left(b_{1}+(1-2 m)\left(\mathcal{N}+2 \alpha^{-}-1\right)\right)
$$

As in the previous case, the scalar potentials $V^{ \pm}$are related by a complex translation:

$$
V^{+}(q)=V^{-}\left(q+K+\mathrm{i} K^{\prime}\right) .
$$


Solvable sectors:

$$
\begin{aligned}
& \mathcal{V}_{\mathcal{N}_{1}}^{ \pm}=(\operatorname{cn} q)^{\alpha^{ \pm}}(\operatorname{sn} q)^{\beta^{ \pm}}(\operatorname{dn} q)^{\beta^{\mp}}\left\langle 1, \operatorname{cn}^{2} q, \ldots,(\operatorname{cn} q)^{2\left(\mathcal{N}_{1}-1\right)}\right\rangle, \\
& \mathcal{V}_{\mathcal{N}_{2}}^{ \pm}=(\operatorname{cn} q)^{1-\alpha^{ \pm}}(\operatorname{sn} q)^{\beta^{ \pm}}(\operatorname{dn} q)^{\beta^{\mp}}\left\langle 1, \operatorname{cn}^{2} q, \ldots,(\operatorname{cn} q)^{2\left(\mathcal{N}_{2}-1\right)}\right\rangle .
\end{aligned}
$$

The potentials are again singular at integer multiples of $K$, so that their Hamiltonians $H^{ \pm}$ can be taken as defined on, e.g., $S=(0, K)$. The conditions ensuring the finiteness of the $L^{2}$ norm in the solvable sectors are given by Eqs. (IV.15).

Case 5. $A(z)=\frac{1}{2} z\left(z^{2}+2(1-2 m) z+1\right)$.

Change of variable: $z=\frac{1+\mathrm{cn} q}{1-\mathrm{cn} q}$.

Supercharge:

$$
\begin{gathered}
E=-\frac{\operatorname{cn} q+2 \operatorname{dn}^{2} q}{\operatorname{sn} q \operatorname{dn} q}, \quad F=-2 \frac{\operatorname{dn} q}{\operatorname{sn} q} \\
W=\frac{\left(2 b_{1}-\mathcal{N}-2 \alpha^{-}+1\right) \operatorname{sn}^{2} q+2\left(\mathcal{N}+2 \alpha^{-}-1\right)}{4 \operatorname{sn} q \operatorname{dn} q} .
\end{gathered}
$$

Potentials:

$$
V^{ \pm}=\frac{\alpha^{ \pm}\left(\alpha^{ \pm}-1\right)}{4(1+\operatorname{cn} q)}+\frac{\alpha^{\mp}\left(\alpha^{\mp}-1\right)}{4(1-\operatorname{cn} q)}+\frac{\mathcal{N} m \beta^{ \pm} \operatorname{cn} q+\left(\beta^{ \pm}\right)^{2}+\frac{1}{4} m m^{\prime}\left(1-\mathcal{N}^{2}\right)}{2 \operatorname{dn}^{2} q}+V_{0} .
$$

Parameters:

$$
\beta^{ \pm}= \pm \frac{1}{4}\left(2 b_{1}-(1-2 m)\left(\mathcal{N}+2 \alpha^{-}-1\right)\right) .
$$

The scalar potentials $V^{ \pm}$are related by a real translation, namely

$$
V^{+}(q)=V^{-}(q+2 K)
$$

Hence $H^{+}$and $H^{-}$are self-isospectral in this case, cf. [28].

Gauge factors:

$$
\mathrm{e}^{-\mathcal{U}^{ \pm}}=\left(\frac{1-\operatorname{cn} q}{\operatorname{dn} q}\right)^{\frac{\mathcal{N}-1}{2}} \exp \left(-\frac{\beta^{ \pm}}{k k^{\prime}} \arctan \frac{k^{2} \operatorname{cn} q+k^{\prime 2}}{k k^{\prime}(1-\operatorname{cn} q)}\right)
$$

where $k^{\prime}=\sqrt{m^{\prime}} \equiv \sqrt{1-m}$.

The potentials $V^{ \pm}$are singular at integer multiples of $2 K$, so that their corresponding Hamiltonians are naturally defined on, e.g., the interval $S=(0,2 K)$. The necessary and sufficient conditions ensuring the square integrability of the wave functions in the solvable sectors are given by

$$
\begin{aligned}
& \mathcal{V}_{\mathcal{N}_{1}}^{ \pm} \subset L^{2}(S) \quad \Longleftrightarrow \quad-\frac{1}{2}<\alpha^{ \pm}<\mathcal{N}_{2}-\mathcal{N}_{1}+\frac{3}{2}, \\
& \mathcal{V}_{\mathcal{N}_{2}}^{ \pm} \subset L^{2}(S) \quad \Longleftrightarrow \quad-\frac{1}{2}+\mathcal{N}_{2}-\mathcal{N}_{1}<\alpha^{ \pm}<\frac{3}{2} .
\end{aligned}
$$

Note, in particular, that the condition $\mathcal{N}_{1} \geq \mathcal{N}_{2}$ implies that the inequalities (IV.29a cannot hold unless $\mathcal{N}_{1}-\mathcal{N}_{2}=0,1$. 


\section{N-FOLD SUPERALGEBRA AND ASSOCIATED POLYNOMIAL FAMILIES}

In ordinary ("one-fold") supersymmetric quantum mechanics, it is well known that the superHamiltonian $\mathbf{H}$ is proportional to the anticommutator of the supercharges $Q_{\mathcal{N}}^{ \pm}$, and hence the operators $Q_{\mathcal{N}}^{ \pm}$and $\mathbf{H}$ span a three-dimensional Lie superalgebra. This cannot possibly be the case in $\mathcal{N}$-fold supersymmetric quantum mechanics (with $\mathcal{N}>1$ ), since the anticommutator

$$
\mathcal{H}_{\mathcal{N}} \equiv \frac{1}{2}\left\{Q_{\mathcal{N}}^{+}, Q_{\mathcal{N}}^{-}\right\}
$$

is now a linear differential operator of order $2 \mathcal{N}$. Note, however, that from the nilpotency of the supercharges (Eq. (II.5) ) and the supersymmetric character of $\mathbf{H}$ (Eq. (II.8)), it immediately follows that the anticommutator (V.1), that we shall henceforth call the mother Hamiltonian, commutes with $Q_{\mathcal{N}}^{ \pm}$and with $\mathbf{H}$, namely

$$
\left[\mathcal{H}_{\mathcal{N}}, Q_{\mathcal{N}}^{ \pm}\right]=\left[\mathcal{H}_{\mathcal{N}}, \mathbf{H}\right]=0
$$

These relations strongly suggest that the mother Hamiltonian $\mathcal{H}_{\mathcal{N}}$ is a polynomial of degree $\mathcal{N}$ in $\mathbf{H}$, say $\mathcal{H}_{\mathcal{N}}=\Pi_{\mathcal{N}}(\mathbf{H})$, and hence that the operators $Q_{\mathcal{N}}^{ \pm}$and $\mathbf{H}$ span a nonlinear superalgebra of degree $\mathcal{N}$ defined by the relations (11.5), (11.8), and

$$
\left\{Q_{\mathcal{N}}^{+}, Q_{\mathcal{N}}^{-}\right\}=2 \Pi_{\mathcal{N}}(\mathbf{H})
$$

That this is indeed the case was rigorously proved in Refs. 11, 2], where it was also shown that $\Pi_{\mathcal{N}}$ is proportional to the characteristic polynomial of the restriction of the component Hamiltonians $H^{ \pm}$to the invariant spaces $\mathcal{V}_{\mathcal{N}}^{ \pm}$, namely

$$
\Pi_{\mathcal{N}}(E)=2^{\mathcal{N}-1} \operatorname{det}\left(\left.H^{ \pm}\right|_{\mathcal{V}_{\mathcal{N}}^{ \pm}}-E\right)
$$

By Eqs. (II.37) and (III.35), this is equivalent to the "gauged" relation

$$
\Pi_{\mathcal{N}}(E)=2^{\mathcal{N}-1} \operatorname{det}\left(\left.\overline{\tilde{H}}^{ \pm}\right|_{\tilde{\mathcal{V}}_{\mathcal{N}}^{ \pm}}-E\right) .
$$

It was later shown in Ref. 14 that for type A models the right-hand side of the latter equation is proportional to the critical generalized Bender-Dunne polynomial (GBDP) associated to the gauged Hamiltonian $\overline{\tilde{H}}^{ \pm}$, which in this case is Lie-algebraic with respect to the Lie algebra $\mathfrak{s l}(2)$. Bender-Dunne polynomials were introduced in Ref. [29] to determine the solvable part of the spectrum of a well-known quasi-exactly solvable sextic oscillator Hamiltonian [6], and were soon generalized by Finkel et al. [30] to virtually all one-dimensional quasi-exactly solvable models associated to the $\mathfrak{s l}(2)$ algebra.

We shall now determine the polynomial $\Pi_{\mathcal{N}}$ for the type $\mathrm{C}$ models constructed in the previous section; in particular, we shall show that in this case $\Pi_{\mathcal{N}}$ factorizes (up to a multiplicative constant) as the product of two critical Bender-Dunne type polynomials of degrees $\mathcal{N}_{1}$ and $\mathcal{N}_{2}$. In view of the equality of the characteristic polynomials of the operators $\left.\tilde{H}^{-}\right|_{\tilde{\mathcal{V}}_{\mathcal{N}}^{-}}$ and $\left.\bar{H}^{+}\right|_{\overline{\mathcal{V}}_{\mathcal{N}}^{+}}$(which follows in general from Eq. (V.5) ), it suffices to study the action of one of the gauged Hamiltonians $\overline{\tilde{H}}^{ \pm}$in its corresponding invariant space, e.g., of $\tilde{H} \equiv \tilde{H}^{-}$in $\tilde{\mathcal{V}}_{\mathcal{N}} \equiv \tilde{\mathcal{V}}_{\mathcal{N}}^{-}$

Recall, to begin with, that the linear space $\tilde{\mathcal{V}}_{\mathcal{N}}$ is the direct sum

$$
\tilde{\mathcal{V}}_{\mathcal{N}}=\left\langle 1, z, \ldots, z^{\mathcal{N}_{1}-1}\right\rangle \oplus z^{\lambda}\left\langle 1, z, \ldots, z^{\mathcal{N}_{2}-1}\right\rangle \equiv \tilde{\mathcal{V}}_{\mathcal{N}_{1}}^{(\mathrm{A})} \oplus \tilde{\mathcal{V}}_{\mathcal{N}_{2}}^{(\mathrm{A})}
$$


of the two spaces $\tilde{\mathcal{V}}_{\mathcal{N}_{i}}^{(\text {A) }}$ (cf. Eq. (III.3) ), each of which is invariant under the action of $\tilde{H}$. Hence

$$
\operatorname{det}\left(\left.\tilde{H}\right|_{\tilde{\mathcal{V}}_{\mathcal{N}}}-E\right)=\operatorname{det}\left(\tilde{H}^{(1)}-E\right) \cdot \operatorname{det}\left(\tilde{H}^{(2)}-E\right)
$$

where we have set

$$
\tilde{H}^{(i)}=\left.\tilde{H}\right|_{\tilde{\mathcal{V}}_{\mathcal{N}_{i}}^{(\mathrm{A})}} .
$$

The second-order operator $z^{-(i-1) \lambda} \tilde{H}^{(i)} z^{(i-1) \lambda}(i=1,2)$ preserves a type A space (III.3) of dimension $\mathcal{N}_{i}$, and the coefficient of $\partial_{z}^{2}$ in this operator is the polynomial $A(z)$. Since $A(z)$ has degree three and vanishes at the origin (see Eq. (III.9a)), it follows 30] that each of the operators $z^{-(i-1) \lambda} \tilde{H}^{(i)} z^{(i-1) \lambda}(i=1,2)$ defines an associated family of GBDPs $\left\{\pi_{i, k}^{\left[\mathcal{N}_{1}, \mathcal{N}_{2}\right]}\right\}_{k=0}^{\infty}$ whose critical element $\pi_{i, \mathcal{N}_{i}}^{\left[\mathcal{N}_{1}, \mathcal{N}_{2}\right]}$ (since $\mathcal{N}_{i}=\operatorname{dim} \tilde{\mathcal{V}}_{\mathcal{N}_{i}}^{(\mathrm{A})}$ ) is proportional to the characteristic polynomial of $z^{-(i-1) \lambda} \tilde{H}^{(i)} z^{(i-1) \lambda}$, and hence of $\tilde{H}^{(i)}$ :

$$
\operatorname{det}\left(\tilde{H}^{(i)}-E\right)=\operatorname{det}\left(z^{-(i-1) \lambda} \tilde{H}^{(i)} z^{(i-1) \lambda}-E\right)=(-1)^{\mathcal{N}_{i}} \pi_{i, \mathcal{N}_{i}}^{\left[\mathcal{N}_{1}, \mathcal{N}_{2}\right]}(E), \quad i=1,2 .
$$

By Eqs. (V.5) and (V.7) we thus have

$$
\Pi_{\mathcal{N}}=(-1)^{\mathcal{N}} 2^{\mathcal{N}-1} \pi_{1, \mathcal{N}_{1}}^{\left[\mathcal{N}_{1}, \mathcal{N}_{2}\right]} \pi_{2, \mathcal{N}_{2}}^{\left[\mathcal{N}_{1}, \mathcal{N}_{2}\right]}
$$

and consequently (cf. Eq. ( $(\overline{V .3})$ ) the type $\mathrm{C} \mathcal{N}$-fold superalgebra is given by

$$
\begin{aligned}
& \left\{Q_{\mathcal{N}}^{ \pm}, Q_{\mathcal{N}}^{ \pm}\right\}=\left[Q_{\mathcal{N}}^{ \pm}, \mathbf{H}\right]=0, \\
& \left\{Q_{\mathcal{N}}^{+}, Q_{\mathcal{N}}^{-}\right\}=(-2)^{\mathcal{N}} \pi_{1, \mathcal{N}_{1}}^{\left[\mathcal{N}_{1} \mathcal{N}_{2}\right]}(\mathbf{H}) \pi_{2, \mathcal{N}_{2}}^{\left[\mathcal{N}_{1}, \mathcal{N}_{2}\right]}(\mathbf{H}) .
\end{aligned}
$$

We should note at this point that, as previously remarked, the operators of the form $z^{-(i-1) \lambda} \tilde{H}^{(i)} z^{(i-1) \lambda}(i=1,2)$ do not exhaust all the possible gauged Hamiltonians of type A. As a consequence, some of the characteristic features of the type A models are inevitably lost in the type $\mathrm{C}$ case. In particular, the $\mathrm{GL}(2, \mathbb{R})$ invariance of type A models, which ensures that all the coefficients of the associated GBDPs are expressed in terms of polynomial invariants [14], is broken in type $\mathrm{C}$ models and, as a consequence, the same is true for the $\mathcal{N}$-fold superalgebra of type $\mathrm{C}$. In other words, the type $\mathrm{C}$ models have two invariant subspaces of type A at the cost of the $\operatorname{GL}(2, \mathbb{R})$ symmetry. On the other hand, it turns out that each of the polynomial families associated with type $\mathrm{C}$ models acquires a novel feature, namely the dependency on two positive integers $\mathcal{N}_{1}$ and $\mathcal{N}_{2}$ (cf. Eq. (V.18) below). That is the reason for the rather cumbersome notation $\pi_{i, k}^{\left[\mathcal{N}_{1}, \mathcal{N}_{2}\right]}$, that we shall hereafter abbreviate as $\pi_{i, k}$ unless the dependence on $\mathcal{N}_{1}$ and $\mathcal{N}_{2}$ is crucial.

To construct the polynomial families $\left\{\pi_{i_{k}}\right\}_{k=0}^{\infty}(i=1,2)$ associated with type $\mathrm{C}$ models, let $\chi_{E}(z)$ denote an eigenfunction of $\tilde{H}$ with eigenvalue $E$. In view of Eq. (V.6), we shall consider the following two formal expansions of this eigenfunction in powers of $z$ :

$$
\chi_{E}(z)=z^{(i-1) \lambda} \sum_{k=0}^{\infty} \frac{\hat{\pi}_{i, k}(E)}{\Gamma(k+1+(i-1) \lambda)} z^{k}, \quad i=1,2 .
$$

Here we have set $\hat{\pi}_{i, k}=\gamma_{i, k} \pi_{i, k}, \gamma_{i, k}$ being a numerical coefficient that must be chosen so that $\pi_{i, k}$ is monic. Clearly, the necessary and sufficient condition for $\chi_{E}(z)$ to belong to $\tilde{\mathcal{V}}_{\mathcal{N}_{i}}^{(\mathrm{A})}$ is that

$$
\hat{\pi}_{i, k}(E)=0, \quad \text { for all } k \geq \mathcal{N}_{i} .
$$


Acting on $\chi_{E}(z)$ with $\tilde{H}$ and using Eqs. (II.14), (III.9), and (III.11) one immediately arrives at the following recursion relation for the coefficients $\hat{\pi}_{i, k}(E)$ :

$$
\begin{aligned}
& a_{1}(k+(i-2) \lambda+1) \hat{\pi}_{i, k+1}= \\
& -\left[E+c_{0}+(k+(i-1) \lambda)\left(b_{1}+a_{2}(k+(i-1) \lambda-\mathcal{N}+1)\right)\right] \hat{\pi}_{i, k} \\
& \quad-a_{3}(k+(i-1) \lambda)[(k+(i-1) \lambda-1)(k+(i-2) \lambda-\mathcal{N}+1) \\
& \left.\quad+\left(\mathcal{N}_{1}-1\right)\left(\mathcal{N}_{2}+\lambda-1\right)\right] \hat{\pi}_{i, k-1} ; \quad k \geq 0, \quad \hat{\pi}_{i,-1} \equiv 0 .
\end{aligned}
$$

Note that the coefficient $a_{1}$ is nonzero in all the canonical forms listed in Table \with the exception of the second one, which corresponds to the trivial case of a constant potential. Hence we shall assume in what follows that $a_{1} \neq 0$. In that case the recurrence relation (V.14) can be brought to the more standard form

$$
\begin{aligned}
& \pi_{i, k+1}=\left[E+c_{0}\right.\left.+(k+(i-1) \lambda)\left(b_{1}+a_{2}(k+(i-1) \lambda-\mathcal{N}+1)\right)\right] \pi_{i, k} \\
&-a_{1} a_{3}(k+(i-1) \lambda)(k+(i-2) \lambda)[(k+(i-1) \lambda-1) \\
& \times\left.(k+(i-2) \lambda-\mathcal{N}+1)+\left(\mathcal{N}_{1}-1\right)\left(\mathcal{N}_{2}+\lambda-1\right)\right] \pi_{i, k-1} ; \\
& k \geq 0, \quad \pi_{i, 0} \equiv 1,
\end{aligned}
$$

by choosing the so far undetermined multipliers $\gamma_{i, k}$ as follows:

$$
\gamma_{i, k}=\frac{1}{\left(-a_{1}\right)^{k} \Gamma(k+1+(i-2) \lambda)}, \quad k \geq 0 .
$$

The three-term recursion relation (V.15) will actually define a family of weakly orthogonal polynomials $\left\{\pi_{i, k}\right\}_{k=0}^{\infty}$ provided that the coefficient of $\pi_{i, k-1}$ vanishes for some non-negative integer value of $k$ [31]. If $k=K$ is the lowest such value, and $E_{j}(j=1, \ldots, K)$ is a root of the critical polynomial $\pi_{i, K}$, then the recursion relation (V.15) implies that $\pi_{i, k}\left(E_{j}\right)=0$ for all $k \geq K$. It follows [29, 30] that the linear space $z^{(i-1) \lambda}\left\langle 1, z, \ldots, z^{K-1}\right\rangle$ is invariant under $\tilde{H}$, and that the eigenvalues of the restriction of $\tilde{H}$ to this space are the $K$ roots (counting multiplicities) of the critical polynomial $\pi_{i, K}$. In particular, the characteristic polynomial of the restriction of $\tilde{H}$ to the invariant space $z^{(i-1) \lambda}\left\langle 1, z, \ldots, z^{K-1}\right\rangle$ is proportional to $\pi_{i, K}(E)$. For the recursion relation ( $(\overline{15})$, the coefficient of $\pi_{i, k-1}$ can be written as

$$
\begin{cases}-a_{1} a_{3} k\left(k-\mathcal{N}_{1}\right)(k-\lambda)\left(k-\mathcal{N}_{2}-\lambda\right), & i=1 \\ -a_{1} a_{3} k\left(k-\mathcal{N}_{2}\right)(k+\lambda)\left(k-\mathcal{N}_{1}+\lambda\right), & i=2 .\end{cases}
$$

Taking into account the restrictions (III.28) on $\lambda$, it is easily seen that the degree of the critical polynomial is $K=\mathcal{N}_{i}, i=1,2$. This establishes Eq. (V.9), since the space $z^{(i-1) \lambda}\left\langle 1, z, \ldots, z^{\mathcal{N}_{i}-1}\right\rangle$ coincides with $\tilde{\mathcal{V}}_{\mathcal{N}_{i}}^{(\mathrm{A})}(i=1,2)$ by Eq. (V.6).

It is worth mentioning that the polynomial systems $\pi_{i, k}$ associated with type $\mathrm{C} \mathcal{N}$-fold supersymmetry are always weakly orthogonal in spite of the fact that the solvable sectors $\mathcal{V}_{\mathcal{N}}^{ \pm}$are not always normalizable, as was studied in the preceding section. We thus obtain further evidence of the claim in Ref. [14] that normalizability has in general nothing to do 
with the weak orthogonality of the associated GBDPs. Note also that Eq. (V.15) becomes a two-term recursion relation if and only if the coefficient $a_{3}$ vanishes, or, equivalently, if the corresponding Hamiltonian is solvable in Turbiner's sense.

The recursion relation (V.15) determining the Bender-Dunne type polynomials $\pi_{i, k}$ and, ultimately, the $\mathcal{N}$-fold superalgebra via Eq. (V.11b), can be recast into the following more concise form using Eq. (V.17):

$$
\begin{aligned}
& \pi_{i, k+1}=\left[E+c_{0}+(k+(i-1) \lambda)\left(b_{1}+a_{2}(k+(i-1) \lambda-\mathcal{N}+1)\right)\right] \pi_{i, k} \\
& -a_{1} a_{3} k\left(k-\mathcal{N}_{i}\right)(k+(2 i-3) \lambda)\left(k-\mathcal{N}_{3-i}+(2 i-3) \lambda\right) \pi_{i, k-1} .
\end{aligned}
$$

This recursion relation can be used without difficulty to compute the polynomial families $\pi_{i, k}^{\left[\mathcal{N}_{1}, \mathcal{N}_{2}\right]}$ and, in particular, the critical polynomials determining the $\mathcal{N}$-fold superalgebra, for any given values of $\mathcal{N}_{1}$ and $\mathcal{N}_{2}$. We shall exhibit in what follows a few examples of these polynomials for $\mathcal{N}=3$ and 4 , assuming that $\mathcal{N}_{1}$ and $\mathcal{N}_{2}$ satisfy the restriction $\mathcal{N}_{1} \geq \mathcal{N}_{2}$ imposed in Section III.

Example 1. $\mathcal{N}_{1}=2, \mathcal{N}_{2}=1$.

Polynomial system:

$$
\begin{aligned}
& \pi_{1,1}^{[2,1]}(E)=E+c_{0}, \\
& \pi_{1,2}^{[2,1]}(E)=\left(E+c_{0}\right)\left(E+c_{0}+b_{1}-a_{2}\right)+\lambda(\lambda-1) a_{1} a_{3}, \\
& \pi_{2,1}^{[2,1]}(E)=E+c_{0}+\lambda b_{1}+\lambda(\lambda-2) a_{2} .
\end{aligned}
$$

3-fold superalgebra:

$$
\left\{Q_{3}^{+}, Q_{3}^{-}\right\}=-8\left[\left(\mathbf{H}+c_{0}\right)\left(\mathbf{H}+c_{0}+b_{1}-a_{2}\right)+\lambda(\lambda-1) a_{1} a_{3}\right]\left[\mathbf{H}+c_{0}+\lambda b_{1}+\lambda(\lambda-2) a_{2}\right] .
$$

Example 2. $\mathcal{N}_{1}=3, \mathcal{N}_{2}=1$.

Polynomial system:

$$
\begin{aligned}
\pi_{1,1}^{[3,1]}(E)= & E+c_{0}, \\
\pi_{1,2}^{[3,1]}(E)= & \left(E+c_{0}\right)\left(E+c_{0}+b_{1}-2 a_{2}\right)+2 \lambda(\lambda-1) a_{1} a_{3}, \\
\pi_{1,3}^{[3,1]}(E)= & \left(E+c_{0}\right)\left(E+c_{0}+b_{1}-2 a_{2}\right)\left(E+c_{0}+2 b_{1}-2 a_{2}\right) \\
& +4(\lambda-1) a_{1} a_{3}\left((\lambda-1)\left(E+c_{0}\right)+\lambda\left(b_{1}-a_{2}\right)\right) \\
& \\
\pi_{2,1}^{[3,1]}(E)= & E+c_{0}+\lambda b_{1}+\lambda(\lambda-3) a_{2} .
\end{aligned}
$$

4-fold superalgebra:

$$
\begin{aligned}
\left\{Q_{4}^{+}, Q_{4}^{-}\right\}= & 16\left[\left(\mathbf{H}+c_{0}\right)\left(\mathbf{H}+c_{0}+b_{1}-2 a_{2}\right)\left(\mathbf{H}+c_{0}+2 b_{1}-2 a_{2}\right)\right. \\
& \left.+4(\lambda-1) a_{1} a_{3}\left((\lambda-1)\left(\mathbf{H}+c_{0}\right)+\lambda\left(b_{1}-a_{2}\right)\right)\right] \\
& \times\left[\mathbf{H}+c_{0}+\lambda b_{1}+\lambda(\lambda-3) a_{2}\right]
\end{aligned}
$$

Example 3. $\mathcal{N}_{1}=2, \mathcal{N}_{2}=2$. 
Polynomial system:

$$
\begin{aligned}
\pi_{1,1}^{[2,2]}(E)= & E+c_{0}, \\
\pi_{1,2}^{[2,2]}(E)= & \left(E+c_{0}\right)\left(E+c_{0}+b_{1}-2 a_{2}\right)+\left(\lambda^{2}-1\right) a_{1} a_{3}, \\
\pi_{2,1}^{[2,2]}(E)= & E+c_{0}+\lambda b_{1}+\lambda(\lambda-3) a_{2} . \\
\pi_{2,2}^{[2,2]}(E)= & \left(E+c_{0}+(\lambda+1) b_{1}+(\lambda+1)(\lambda-2) a_{2}\right) \\
& \times\left(E+c_{0}+\lambda b_{1}+\lambda(\lambda-3) a_{2}\right)+\left(\lambda^{2}-1\right) a_{1} a_{3} .
\end{aligned}
$$

4-fold superalgebra:

$$
\begin{aligned}
\left\{Q_{4}^{+}, Q_{4}^{-}\right\}= & 16\left[\left(\mathbf{H}+c_{0}\right)\left(\mathbf{H}+c_{0}+b_{1}-2 a_{2}\right)+\left(\lambda^{2}-1\right) a_{1} a_{3}\right] \\
& \times\left[\left(\mathbf{H}+c_{0}+(\lambda+1) b_{1}+(\lambda+1)(\lambda-2) a_{2}\right)\right. \\
& \left.\times\left(\mathbf{H}+c_{0}+\lambda b_{1}+\lambda(\lambda-3) a_{2}\right)+\left(\lambda^{2}-1\right) a_{1} a_{3}\right] .
\end{aligned}
$$

\section{SUMMARY AND DISCUSSION}

In this article we develop an algorithmic procedure for constructing an $\mathcal{N}$-fold supersymmetric quantum system starting from a given finite-dimensional space of functions invariant under a suitable gauge transform of one of the supercharges. Although the method is very general, it is especially useful when one knows a particular example of quasi-solvable Hamiltonian and its algebraically solvable wave functions. We have applied this procedure to the monomial spaces of Post-Turbiner type, thus obtaining the new models of type $\mathrm{C}$ as well as recovering the previously known type $\mathrm{A}$ and type $\mathrm{B}$ systems. We would also like to stress that, although the procedure always yields an $\mathcal{N}$-fold supersymmetric system $\left(H^{ \pm}, P_{\mathcal{N}}\right)$, it does not rule out the existence of more general systems of the same type. For instance, for the type $\mathrm{C}$ models specifically discussed in this paper the supercharges are given by Eqs. (II.3) and (III.22), and the method developed in this paper guarantees that the supersymmetry algebra holds for suitable Hamiltonians $H^{ \pm}$provided that the functions $E$ and $F$ satisfy Eq. (III.18). It is not clear, however, whether this sufficient condition is also necessary. It should also be noted in this respect that the situation is completely analogous for the type B models discussed in Ref. [15].

The normalizability of the solvable sectors of the type $\mathrm{C}$ models, which plays an important role for the existence of dynamical $\mathcal{N}$-fold supersymmetry breaking [1, 12], is briefly investigated in Section IV] From Eqs. (IV.6), (IV.15), (IV.18), and (IV.29), we see that there is little chance for both sectors $\mathcal{V}_{\mathcal{N}_{1}}^{+}$and $\mathcal{V}_{\mathcal{N}_{2}}^{+}\left(\right.$resp. $\mathcal{V}_{\mathcal{N}_{1}}^{-}$and $\mathcal{V}_{\mathcal{N}_{2}}^{-}$) to be simultaneously normalizable. This means that, in general, only a part of the whole solvable sector $\mathcal{V}_{\mathcal{N}}^{+}$ (resp. $\mathcal{V}_{\mathcal{N}}^{-}$) is physical in type $\mathrm{C} \mathcal{N}$-fold supersymmetric systems. For example, among the four subsectors $\mathcal{V}_{\mathcal{N}_{i}}^{ \pm}$in Case 1 only $\mathcal{V}_{\mathcal{N}_{2}}^{-}$is normalizable and thus physical (boundary conditions aside) if $b_{1}<0$ and $\lambda \geq 1$. We can thus say that $\mathcal{N}$-fold supersymmetry is partially broken in type $\mathrm{C}$ models. This phenomenon is novel for type $\mathrm{C}$ due to the characteristic structure of its solvable sector, and, to the best of the authors' knowledge, has not been previously mentioned in the literature. For a more precise discussion, we need of course to deal with the boundary conditions at the singularities in order to define a self-adjoint extension of the Hamiltonians. This kind of mathematical subtlety is beyond the scope of the present 
article, and we shall therefore content ourselves with referring the reader to, e.g, Ref. 32] for a recent discussion of this topic. We also note, in this connection, that the significance of the boundary conditions in (ordinary) supersymmetry breaking was recently reported in a different context, namely, through the careful calculation of the fermion determinant arising out of the path integral formalism [33].

The structure of the solvable sectors in type $\mathrm{C}$ models gives rise to another interesting phenomenon in the theory of exact solutions of the Schrödinger equation. Indeed, when the parameter $\alpha^{+}$(resp. $\alpha^{-}$) is a positive integer $l+1$ (in both cases $\lambda$ is a half-integer, cf. Eq. (III.33) $)$, the potential $V^{+}$(resp. $V^{-}$) in Case 1 given by Eq. (IV.4) is nothing but the radial harmonic oscillator plus the centrifugal potential with a properly quantized angular momentum $l$. It is then apparent that for $b_{1}>0$ (resp. $b_{1}<0$ ) the first solvable sector $\mathcal{V}_{\mathcal{N}_{1}}^{+}$(resp. $\left.\mathcal{V}_{\mathcal{N}_{1}}^{-}\right)\left(\mathcal{N}_{1}=1,2, \ldots\right)$ in Eq. (IV.5a) contains the physical solutions around $q=0$, while the second sector $\mathcal{V}_{\mathcal{N}_{2}}^{+}$(resp. $\mathcal{V}_{\mathcal{N}_{2}}^{-}$) in Eq. (IV.5b) yields the "second" solutions, usually discarded as unphysical due to their singularity ${ }^{5}$ at the origin.

By Eq. (III.36), we may in fact say that type $\mathrm{C}$ models are characterized by the fact that both linearly independent solutions around $z(q)=0$ are quasi-solvable. More precisely, from Eqs. (II.45), (II.9c), (III.10), (II.16), and Table I it is easily seen that the operator $\tilde{H}_{\mathcal{N}}^{-}-E$ (resp. $\bar{H}_{\mathcal{N}}^{+}-E$ ) has a regular singularity at $z=0$, so that Fuchs's theorem applies. Except in the trivial Case 2, which we shall henceforth ignore, for all values of the energy $E$ the roots of the indicial equation are 0 and $\lambda$ (resp. $\mathcal{N}_{2}$ and $\mathcal{N}_{2}+\bar{\lambda}$ ). From Eqs. (III.13) for the space $\tilde{\mathcal{V}}_{\mathcal{N}}^{-}$, and the analogous decomposition

$$
\overline{\mathcal{V}}_{\mathcal{N}}^{+}=z^{\mathcal{N}_{2}}\left\langle 1, \ldots, z^{\mathcal{N}_{1}-1}\right\rangle \oplus z^{\mathcal{N}_{2}+\bar{\lambda}}\left\langle 1, \ldots, z^{\mathcal{N}_{2}-1}\right\rangle
$$

for $\overline{\mathcal{V}}_{\mathcal{N}}^{+}$, we see that each of the four sectors of the invariant spaces $\overline{\tilde{V}}_{\mathcal{N}}^{ \pm}$consists of the solvable eigenfunctions of $\overline{\tilde{H}}_{\mathcal{N}}^{ \pm}$behaving as $z^{i}$ near $z=0$, where $i$ is one of the roots of the indicial equation listed above. Note, in this respect, that this remark is still valid when the difference of the roots of the indicial equation, which is given by $\lambda$ (resp. $\bar{\lambda}$ ), is an integer, even if in this case Fuchs's theorem can only guarantee the existence of one linearly independent power series solution around $z=0$.

The construction of the Bender-Dunne type polynomial systems associated with the type $\mathrm{C}$ models turned out to be straightforward. The breakdown of the $\operatorname{GL}(2, \mathbb{R})$ symmetry in type $\mathrm{C}$ models spoils the characteristic feature possessed by the type A polynomials. Instead, the GBDPs of type $\mathrm{C}$ have a novel dependence on two positive-integer parameters $\mathcal{N}_{1}$ and $\mathcal{N}_{2}$. For any given pair $\left(\mathcal{N}_{1}, \mathcal{N}_{2}\right) \in \mathbb{N} \times \mathbb{N}$, we obtain two related families of weakly orthogonal polynomials satisfying the recursion relation (V.18). The assertion in Ref. 14] that normalizability has nothing to do with the weak orthogonality of the associated family of polynomials has also been confirmed. We also note that a similar construction for the type B systems presents considerable difficulties due to the lack of form-invariance under projective transformations of these models. This difficulty has also prevented the systematic calculation of the explicit form of the type B $\mathcal{N}$-fold superalgebra.

All the quasi-solvable second-order differential operators in one variable preserving a finite-dimensional linear space of monomials, which were classified in Ref. [16], have now been brought into the framework of $\mathcal{N}$-fold supersymmetry. ${ }^{6}$ The natural continuation of

\footnotetext{
${ }^{5}$ Or their non-vanishing, for $l=0$.

${ }^{6}$ With only a few exceptions for $\mathcal{N}=3$ and $\mathcal{N}=4$.
} 
the present work is the study of operators possessing non-monomial type invariant subspaces, and the construction of the associated supersymmetric models following the general algorithm developed in Section [II. It would also be of great interest to extend the results obtained in this paper to a multi-dimensional space-time. Indeed, the generalization of $\mathcal{N}$ fold supersymmetry to higher-dimensional space-times remains one of the most challenging open problems in this field. Recently, Smilga showed [34] that some $\mathcal{N}$-fold supersymmetric systems with $\mathcal{N}=2$ can be realized as weakly supersymmetric field theories in one spacetime dimension. Although the results in Ref. [34] clearly indicate the existence of rather severe obstructions, further investigation in this direction would still be certainly worth undertaking.

\section{Acknowledgments}

This work was partially supported by Spain's DGI under grant no. BFM2002-02646 (A. G.-L.), as well as by a Spanish Ministry of Education, Culture and Sports research fellowship (T. T.).

[1] Aoyama H, Sato $M$ and Tanaka $T 2001 \mathcal{N}$-fold supersymmetry in quantum mechanics: general formalism Nucl. Phys. B 619 105-127 (quant-ph/0106037)

[2] Andrianov A and Sokolov A 2003 Nonlinear supersymmetry in quantum mechanics: algebraic properties and differential representations Nucl. Phys. B 660 25-50 (hep-th/0301062)

[3] Darboux G 1882 Sur une proposition relative aux équations linéaires Comptes Rendus Acad. Sci. 94 1456-1459

[4] Matveev V and Salle M 1991 Darboux Transformations and Solitons (Berlin: Springer Verlag)

[5] Andrianov A, Ioffe M and Spiridonov V 1993 Higher-derivative supersymmetry and the Witten index Phys. Lett. A 174 273-279 (hep-th/9303005)

[6] Turbiner A and Ushveridze A 1987 Spectral singularities and quasi-exactly solvable quantal problem Phys. Lett. A 126 181-183

[7] Ushveridze A 1994 Quasi-exactly Solvable Models in Quantum Mechanics (Bristol: IOP Publishing)

[8] Rubakov V and Spiridonov V 1988 Parasupersymmetric quantum mechanics Mod. Phys. Lett. A 3 1337-1347

[9] Beckers J and Debergh N 1990 Parastatistics and supersymmetry in quantum mechanics Nucl. Phys. B $\mathbf{3 4 0} 767-776$

[10] Khare A 1993 Parasupersymmetry in quantum mechanics J. Math. Phys. 34 1277-1294

[11] Durand S 1993 Fractional supersymmetry and quantum mechanics Phys. Lett. B 312 115-120 (hep-th/9305128)

[12] Tanaka T $2003 \mathcal{N}$-fold supersymmetry and quasi-solvability (preprint), to appear in Progress in Mathematical Physics Research (New York: Nova Science Publishers)

[13] Aoyama H, Sato M and Tanaka T 2001 General forms of a $\mathcal{N}$-fold supersymmetric family Phys. Lett. B $\mathbf{5 0 3} 423-429$ (quant-ph/0012065)

[14] Tanaka T 2003 Type A $\mathcal{N}$-fold supersymmetry and generalized Bender-Dunne polynomials Nucl. Phys. B 662 413-446 (hep-th/0212276) 
[15] González-López A and Tanaka T 2004 A new family of $\mathcal{N}$-fold supersymmetry: type B Phys. Lett. B 586 117-124 (hep-th/0307094)

[16] Post G and Turbiner A 1995 Classification of linear differential operators with invariant subspace in monomials Russ. J. Math. Phys. 3 113-122 (funct-an/9307001)

[17] Tanaka T $2004 \mathfrak{s l}(M+1)$ construction of quasi-solvable quantum $M$-body systems Ann. Phys. 309 239-280 (hep-th/0306174)

[18] Shifman M 1989 New findings in quantum mechanics (partial algebraization of the spectral problem) Int. J. Mod. Phys. A 4 2897-2952

[19] González-López A, Kamran N and Olver P J 1994 Quasi-exact solvability Contemp. Math. $160113-140$

[20] Crum M 1955 Associated Sturm-Liouville equations Quart. J. Math. Oxford 6 121-127

[21] Krě̌n M 1957 Dokl. Akad. Nauk SSSR 113970

[22] González-López A, Kamran N and Olver P J 1993 Normalizability of one-dimensional quasiexactly solvable Schrödinger operators Commun. Math. Phys. 153 117-146

[23] Turbiner A 1994 Lie algebras, cohomologies, and new findings in quantum mechanics Contemp. Math. 160 263-310

[24] Turbiner A 1988 Quasi-exactly solvable problems and $\mathfrak{s l}(2)$ algebra Commun. Math. Phys. $118467-474$

[25] Turbiner A 1992 Lie algebras and polynomials in one variable J. Phys. A: Math. Gen. 25 L1087-L1093

[26] Cooper F, Khare A and Sukhatme U 1995 Supersymmetry and quantum mechanics Phys. Rep. 251 267-385 (hep-th/9405029)

[27] Gradshteyn I and Ryzhik I 2000 Table of Integrals, Series, and Products (San Diego: Academic Press) sixth edition

[28] Dunne G and Feinberg J 1998 Self-isospectral potentials and supersymmetric quantum mechanics Phys. Rev. D 57 1271-1276 (hep-th/9706012)

[29] Bender C M and Dunne G V 1996 Quasi-exactly solvable systems and orthogonal polynomials J. Math. Phys. 37 6-11 (hep-th/95111389)

[30] Finkel F, González-López A and Rodríguez M Á 1996 Quasi-exactly solvable potentials on the line and orthogonal polynomials J. Math. Phys. 37 3954-3972 (hep-th/9603103)

[31] Chihara T 1978 An Introduction to Orthogonal Polynomials (New York: Gordon and Breach)

[32] Tsutsui I, Fülöp T and Cheon T 2003 Connection conditions and the spectral family under singular potentials J. Phys. A: Math. Gen. 36 275-287 (quant-ph/0209110)

[33] Kikuchi H 2003 The fermion determinant, its modulus and phase Phys. Lett. B 562 299-306 (hep-th/0210003)

[34] Smilga A 2004 Weak supersymmetry Phys. Lett. B 585 173-179 (hep-th/0311023) 